\title{
Colorectal Cancer: From Genetic Landscape to Targeted Therapy
}

\author{
Mouade El Bali (D), Joaira Bakkach $(\mathbb{D}$, and Mohcine Bennani Mechita \\ Biomedical Genomics and Oncogenetics Research Laboratory, Faculty of Sciences and Techniques of Tangier, \\ Abdelmalek Essaâdi University, Tetouan, Morocco
}

Correspondence should be addressed to Mouade El Bali; mouade.elbali@etu.uae.ac.ma

Received 10 March 2021; Accepted 25 June 2021; Published 6 July 2021

Academic Editor: Alamgeer Yuchi

Copyright ( $\odot 2021$ Mouade El Bali et al. This is an open access article distributed under the Creative Commons Attribution License, which permits unrestricted use, distribution, and reproduction in any medium, provided the original work is properly cited.

Colorectal cancer (CRC) is the third most common cancer type and the second cause of death worldwide. The advancement in understanding molecular pathways involved in CRC has led to new classifications based on the molecular characteristics of each tumor and also improved CRC management through the integration of targeted therapy into clinical practice. In this review, we will present the main molecular pathways involved in CRC carcinogenesis, the molecular classifications. The anti-VEGF and antiEGFR therapies currently used in CRC treatment and those under clinical investigation will also be outlined, as well as the mechanisms of primary and acquired resistance to anti-EGFR monoclonal antibodies (cetuximab and panitumumab). Targeted therapy has led to great improvement in the treatment of metastatic CRC. However, there has been variability in CRC treatment outcomes due to molecular heterogeneity in colorectal tumors, which underscores the need for identifying prognostic and predictive biomarkers for CRC-targeted drugs.

\section{Introduction}

Colorectal cancer (CRC) is considered the third most prevalent cancer and the second cause of death by cancer worldwide [1]. In 2018, 1.8 million new CRC cases were reported and 881,000 persons died of the disease, which accounted for $6.1 \%$ and $9.2 \%$ of new cases and deaths, respectively [2]. An increasing incidence trend of 2.5 million cases has been predicted in 2035 [3].

Currently, the 5-year overall survival (OS) rate of CRC is estimated at $64 \%$ for all stages in the United States, and this seems to decrease to nearly $12 \%$ for metastatic CRC (mCRC) $[4,5]$.

Surgery alone or in combination with chemotherapy and radiotherapy in the adjuvant setting remains the main treatment option in cases of early diagnosis, while surgery is no longer effective for advanced stages that represent $25 \%$ of CRCs cases [6]. Unfortunately, the efficacy of cytotoxic therapies may be altered by the rapid evolution of drug resistance and the occurrence of cancer recurrence [7]. Hence, developing other treatment options for CRC, especially for mCRC to increase its overall survival and reduce its severity, is highly needed.
With the advancement in our understanding of carcinogenesis mechanisms and the underlying molecular pathways, treatment of CRC, especially mCRC, has evolved considerably over the past years, which was reflected by using many chemotherapy combinations and integrating novel targeted drugs into clinical practice. This advancement in chemotherapy and targeted drugs has led to significantly improve OS to over 40 months for mCRC patients [8].

Cetuximab was the first targeted agent for CRC that has been approved by the Food and Drug Administration (FDA) in 2004 , followed by bevacizumab in the same year. Since then, many other targeted drugs for CRC have been brought to market successively (Figure 1). Targeted agents currently used for the treatment of CRC may be divided into three categories: anti-Vascular Endothelial Growth Factor (VEGF) such as bevacizumab, aflibercept, and ramucirumab; anti-Epidermal Growth Factor Receptor (EGFR) antibodies such as cetuximab and panitumumab; and finally multikinase inhibitors like regorafenib [9].

The advancement in understanding molecular pathways involved in CRC carcinogenesis has also led to many molecular classification systems. The Cancer Genome Atlas (TCGA) and the Consensus Molecular Subtype (CMS) are 


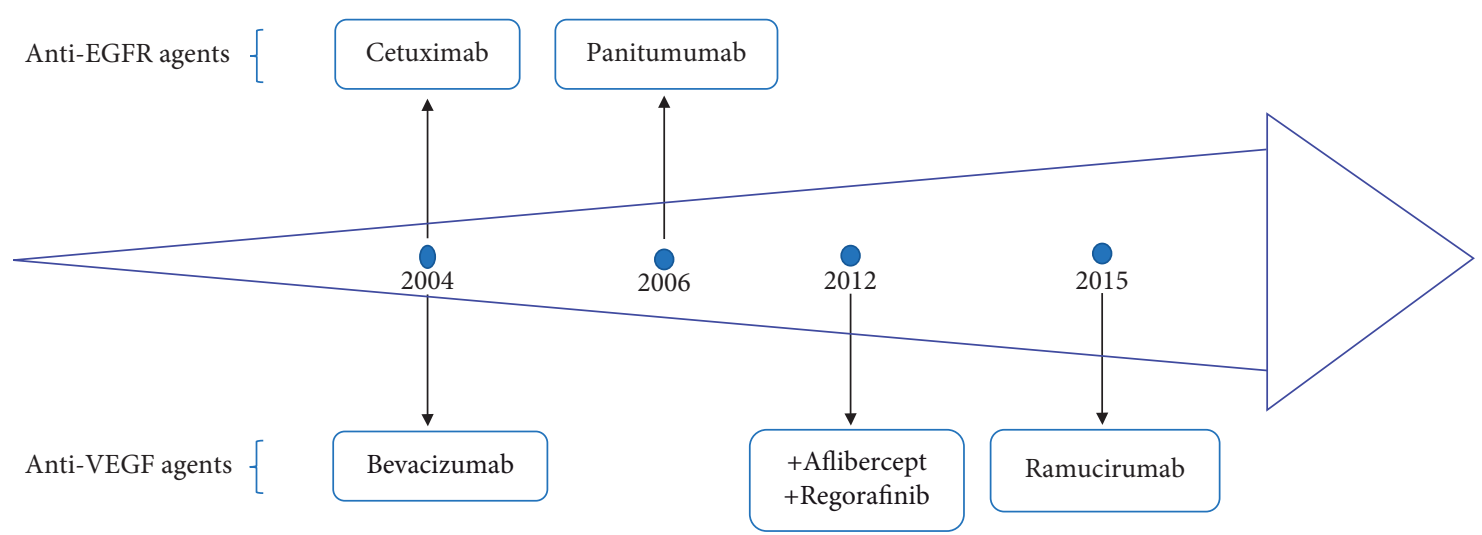

FIGURE 1: Targeted agent approved for colorectal cancer. EGFR: epidermal growth factor receptor; VEGF: vascular endothelial growth factor.

considered the main classifications. CMS classification has been proposed after analyzing the pathological and molecular profile of CRC patients from many studies. In addition to its potential prognostic and predictive value, CMS can also help to explain the CRC heterogeneity caused by genetic and epigenetic mechanisms [10].

The current review aims to provide an overview of molecular pathways involved in CRC carcinogenesis, as well as molecularly defined subtypes and their clinical implication. We will also summarize available and future CRCtargeted agents and discuss anti-EGFR resistance mechanisms.

\section{Molecular Pathways in CRC}

2.1. Inherited CRC. The etiologies of CRC are either genetic or environmental or both [11]. CRC is divided into hereditary and sporadic forms, with approximately $75-80 \%$ of sporadic forms [12].

Approximately $5 \%$ of all CRC cases are caused by inherited germline mutations in some key genes, leading to colorectal carcinogenesis. Approximately $20 \%$ of the remaining $95 \%$ of CRC cases present a positive family history, which cannot account as a true hereditary form of CRC. Many syndromes have been identified; the most frequent syndromes are Lynch Syndrome (Hereditary Nonpolyposis Colorectal Cancer (HNPCC)) and Familial Adenomatous Polyposis (FAP) $[13,14]$.

Lynch Syndrome (HNPCC) is the most common hereditary CRC syndrome representing 2-3\% of all CRC patients [14]. It is an autosomal dominant syndrome, caused by germline mutations in DNA mismatch repair (MMR) genes, which lead to amplifying the replication errors, increasing rate, and the potential of malignancy. These genes included $M L H 1, M S H 2, P M S 2$, and MSH6 resulting in microsatellite instability (MSI) when mutated [15].

Familial Adenomatous Polyposis (FAP) is an autosomal dominant disorder, which is characterized by colorectal adenomatous polyps, which ranged from hundreds to thousands of polyps [16]. It is caused by germline mutations in the adenomatous polyposis coli $(A P C)$ gene with a frequency of $1 \%$ of all CRCs [14]. APC gene is a tumor suppressor gene, coding for a protein that regulates the cytoplasm degradation of $\beta$-catenin. These two molecules are essential components of the Wnt signaling pathway [17].

There are other rare forms of familial CRC including the MYH-Associated Polyposis (MAP), which is an autosomal recessive disorder caused by biallelic mutations in the $M Y H$ gene. Tumors in this form are commonly microsatellite stable and exhibit a high frequency of APC somatic mutations and a low rate of loss of heterozygosity (LOH) [14]. Additionally, the Hamartomatous polyposis syndromes that englobe the syndrome of PeutzJeghers (PJS), the syndrome of Juvenile Polyposis (JPS), and the syndrome of Cowden are autosomal dominant syndromes caused by germline mutations in STK11/ LKB1, BMPR1A/SMAD4, and PTEN, respectively [18].

More details about these hereditary CRC syndromes, diagnosis, and management approaches have been well reviewed by Kastrinos and Syngal [19].

2.2. Sporadic CRC. With respect to sporadic CRC, Fearon et al. have suggested a colorectal carcinogenesis model that correlated specific genetic landscapes with changing tissue morphology, from adenomas to carcinomas $[12,20]$. Genomic instability is considered an essential component of this transformation process [14]. There are three main categories of genomic instability in CRC. Chromosomal instability (CIN), the most frequent representing $70-85 \%$ of CRCs, is characterized by the accumulation of numerical or structural chromosomal abnormalities (aneuploidy). FAP is the inherited syndrome associated with these changes $[21,22]$. Another type of genomic instability is the MSI, which is caused by MMR alteration [22]. With the increasing knowledge with regard to the involvement of epigenetic factors, particularly the promoter sequence methylation, in the development of certain subsets of cancers and polyps, the third pathway of genomic instability has emerged " $\mathrm{CpG}$ Island Methylator Phenotype (CIMP+)" [22]. All these alterations are presented in detail hereinafter.

2.2.1. Chromosome Instability. CIN accounts for $65-70 \%$ of sporadic CRCs. It is characterized by the high frequency of $\mathrm{LOH}$, subchromosomal genomic amplifications, and 
extensive chromosome imbalances (aneuploidy). In addition to these karyotypic abnormalities, the accumulation of particular mutations in some specific tumor suppressor genes and oncogenes activates CRC pathogenesis [23]. The main genes which are mutated during this pathway are as follows:

$A P C$ gene: It is a tumor suppressor gene located on chromosome $5 \mathrm{q} 21$. APC functional protein plays an important role in regulating differentiation, adhesion, apoptosis, development, migration, and chromosomal segregation. Mutations or loss of this gene have been found in $40-70 \%$ of CRCs and were considered the earliest genetic events in colorectal carcinogenesis $[24,25]$.

APC protein is a part of a complex that phosphorylates the $\beta$-catenin, which causes its ubiquitination and destruction in the proteosome. Truncated APC protein destabilizes the complex and increases the cytoplasmic $\beta$-catenin level, which translocates to the nucleus and activates the transcription of various genes involved in tumor growth and invasion, by interacting with the T-cell factor/lymphoid [24]. Half of CRC cases with intact $A P C$ have activating mutations in the $\beta$-catenin gene [26], which reflects the importance of the Wnt pathway.

KRAS oncogene: The frequency of KRAS protooncogene mutations is estimated to range from 30 to $60 \%$ in CRCs and large adenomas. Most activating mutations were found to be located in codons 12 and 13 of exon 1 [27]. Activation of K-Ras is known to affect various cellular pathways that regulate cellular growth, survival, proliferation, apoptosis, cytoskeleton organization, cell motility, differentiation, and inflammation $[14,23]$.

Activation of the KRAS gene has been suggested to play a significant role in the transition from adenoma to carcinoma [28].

SMAD2, SMAD4, and DCC: These three genes are located at chromosome 18q21. The allelic loss of this site has been found in 60\% of CRCs [29]. DCC gene encodes for a transmembrane receptor that promotes apoptosis, whereas SMAD2 and SMAD4 are part of the transforming growth factor- $\beta$ (TGF- $\beta$ ) signaling pathway, which regulates growth as well as apoptosis $[22,30]$.

TP53: It is located on $17 \mathrm{p} 13.1$ and encodes for a tumor suppressor protein $\mathrm{p} 53$ whose inactivation is usually a late event in the CRC carcinogenesis process [31]. It is widely known that p53 dysfunction is a universal biomarker of human tumors and the loss of its function has been reported in $4-26 \%$ of adenomas, $50 \%$ of adenomas with invasive foci, and $50-75 \%$ of CRCs, which define its role in the transition from adenoma to carcinoma $[23,32]$.

The CIN pathway is related to mutations in the APC gene or allelic loss at chromosome 5q (APC, MCC genes), followed by Kirsten rat sarcoma viral oncogene (KRAS) mutation, loss of 18q (DCC, SMAD2, and SMAD4 genes), and finally, deletion of $17 \mathrm{p}$, containing the famous tumor suppressor gene TP53 [24].

2.2.2. Microsatellite Instability. Microsatellites are short sequences with repeated nucleotides, which are distributed across the entire human genome, and consist of mononucleotide, dinucleotide, or higher-order nucleotide repeats such as (A)n or (CA)n [33]. These microsatellites are especially motifs of mutation accumulations, due to the decreasing of DNA polymerase efficiency. The most common errors associated with microsatellites are base-base mismatches and insertion-deletion loops (IDLs) [33, 34].

MMR systems are charged to maintain genomic stability by identifying and repairing base-pair mismatches that occur during DNA replication. Mutator phenotype accompanied with MSI is a result of the MMR systems' inability to correct these errors. There are at least 7 proteins in the mismatch repair systems: hMLH1, hMLH3, hMSH2, hMSH3, hMSH6, hPMS1, and hPMS2, forming 5 protein dimers, which are the MutS $\alpha$ (MSH2, MSH6), MutS $\beta$ (MSH2, MSH3), MutL $\alpha$ (MLH1, PMS2), MutL $\beta$ (MLH1, PMS1), and finally MutL $\gamma$ (MLH1, MLH3) [34, 35].

In order to test the MSI, there are two main methods: immunohistochemistry (IHC) which serves for the detection of the expression level of the four main MMR proteins (MSH2, MSH6, MLH1, and PMS2) directly from the tumor tissue. Loss of expression of at least one of these proteins means that the tumor is deficient MMR (dMMR) and as a consequence MSI [36]. The second method is based on testing a DNA microsatellite panel. In this method, MSI-High (MSI-H) is defined when $40 \%$ of the markers are unstable [36]. In 1998, a panel of five microsatellite markers called the Bethesda panel has been proposed for the first time by the "International Workshop on Microsatellite Instability and RER Phenotypes in Cancer Detection and Familial Predisposition," which includes two mononucleotides (BAT25 and BAT26) and three dinucleotides (D5S346, D2S123, and D17S250) [37]. Another panel called the Pentaplex panel, composed of five mononucleotides markers, has been proposed (BAT25, BAT26, NR21, NR24, and NR27), due to the high sensitivity of mononucleotides markers compared to dinucleotides. Based on the Pentaplex panel, two types of tumors have been established: MSI (MSI-High) with at least three unstable markers, and microsatellite stability (MSS) with no instability, or the instability in one marker [36].

Approximately $15 \%$ of CRC patients show an MSI, $3 \%$ of which are caused by germline mutations (Lynch Syndrome), and $12 \%$ are due generally to sporadic hypermethylation in the promoter of the MLH1 gene [38]. Most studies suggested that MSS tumors had a worse prognosis than those with MSI. Additionally, a strong correlation has been found between sporadic MSI and the existence of V600E BRAF mutation $[39,40]$.

2.2.3. CpG Island Methylator Phenotype. Epigenetic regulation of gene expressions is defined as heritable changes without any alteration in the DNA sequence. These epigenetic changes are found to play an important role in the 
carcinogenesis of many carcinomas including CRC and offer an explanation of some phenotypes of this disease. Histone modifications or DNA methylation are thought to be the most common cause of epigenetic alterations [41].

$\mathrm{CpG}$ island methylation is a typical epigenetic event in colorectal carcinogenesis, accounting for $20 \%$ in CRCs [42]. The concept of CIMP in CRC has been originally reported in 1999 by Toyota et al. [43]. It occurs generally by DNA hypermethylation at the $5^{\prime}$-CG-3' $(\mathrm{CpG})$ dinucleotide in the promoter region, resulting in gene silencing and the function loss of some tumor suppressor genes such as $M L H 1$, $A P C, M C C, M G M T$, and several others [44]. As the CIMP refers to the presence of multiple hypermutated genes, Weisenberger et al. have proposed a panel of five markers, which are CACNA1G, IGF2, NEUROG, RUNX3, and SOCS1. CIMP+ was defined by the methylation of 3 to 5 markers and CIMP- by the hypermethylation of 0 to 2 loci [45]. Other studies classified tumors to CIMP-high (CIMPH) and CIMP-low (CIMP-L) or CIMP-0 [46].

Clinically, CIMP-H CRCs have been associated with female sex, older age, right-sided tumor location, and advanced stage. At the pathologic level, CIMP-H tumors showed higher rates of tumor-infiltrating lymphocytes, Crohn-like infiltrates, perineural, lymphovascular invasion, and higher levels of Fusobacterium nucleatum. With regard to molecular characteristics, this tumor subtype was shown to exhibit a higher prevalence of BRAF and PIK3CA mutation (OR: 20.17 (95\% CI: 13.54-30.05); 1.61 (95\% CI: 1.24-2.10), respectively) and more likely to have higher MSI status (OR: 10.95 (95\% CI: 8.49-14.13)). Additionally, there was an inverse association of CIMP-H tumors with TP53 and KRAS mutations, and no association has been reported with APC mutation [42].

Why CIMP is consistently associated with BRAF mutations has long been a debated question. In 2019, Tao et al. provided compelling evidence that solved this long-standing question suggesting that through the aging-like acquisition of DNA methylation, BRAF mutated cells may survive by suppressing senescence and activating stem cell pathways [47].

\section{Molecular Classification of CRCs and Associated Features}

3.1. Molecular Subtypes of CRC. As we reviewed earlier, there are three major mechanisms of genetic instability in CRC: CIN, MSI, and CIMP. Many studies have tried to establish a molecular classification for CRCs, but these did not lead to a single systematic classification [48].

A systematic molecular pathological classification has been proposed by The Cancer Genome Atlas (TCGA) in 2012 [49] and another one by the Consensus Molecular Subtype (CMS) Consortium in 2015 [50].

Despite the heterogeneity of CRCs, the ancient classification by TCGA has divided CRCs into two subtypes, which are characterized by a specific morphology and molecular alteration.

(i) The hypermutated cancers, representing $16 \%$ of CRCs. Three-quarters of this group have a high MSI as a result of $\mathrm{dMMR}$, and the other one-quarter corresponds to ultramutated cancers with polymerase- $\varepsilon$ (POLE) mutations.

(ii) The nonhypermutated cancers, which account for $84 \%$ of CRC cases. The tumors of this group are MSS, which harbors a higher frequency of alterations in somatic DNA and common mutations in $A P C$, TP53, KRAS, SMAD4, and PIK3CA genes [48, 49].

In 2015, an international consortium has analyzed a large-scale data sharing, aiming to establish a new and universal molecular classification and facilitate its clinical implication. The panel experts evaluated six CRC subtyping algorithms from six studies [51-56] and also the data of TCGA, to develop a novel classification of four CMS groups (Figure 2) [50].

(i) CMS1 (microsatellite instability immune, 14\%): Almost all patients with MSI were regrouped in this group, characterized by hypermutated profile, especially in $M L H 1$ gene, and high level of $B R A F$ mutations. CMS1 patients have a strong immune activation, reflected by a high level in gene expression, associated with a diffuse immune infiltrate and upregulation in immune response pathways (PD1 activation, NK cells, Th1 cells, and cytotoxic T-cell infiltration signatures) [50]. This immune activation is a new feature of MSI CRCs [57]

(ii) CMS2 (canonical, 37\%): It includes patients with higher CIN, which have a high level of somatic copy number alterations (SCNAs). Conversely to CMS1, CMS2 showed a strong upregulation of WNT and MYC downstream targets and epithelial differentiation. Compared with other groups, CMS2 exhibits more frequently copy number gain in oncogenes and copy number losses in tumor suppressor genes [50]

(iii) CMS3 (metabolic,13\%): It is characterized by dysregulation of many metabolic pathways (glucose pentose, nitrogen, fatty acid, etc.), CIN with fewer SCANs, higher prevalence of CIMP-low, and higher KRAS mutations compared with other groups. Almost 30\% of CMS3 was hypermutated, which results in more MSI samples compared with CMS2 and CMS4 [50]

(iv) CMS4 (mesenchymal, 23\%): Higher CIN and increased level of SCNAs, with strong expression of epithelial-to-mesenchymal transition (EMT) genes and activation of TGF- $\beta$ signaling. CMS4 overexpresses more commonly proteins that are implicated in stromal infiltration and angiogenesis and exhibits higher expression level of mesenchymal protein pathways [50]

Finally, there were tumors with mixed features (13\%) that possibly represent either a transition phenotype or intratumoral heterogeneity [50].

3.2. Clinical and Prognostic Associations of the CMSs. CMS1 has been found to be significantly more common in females with higher histological grades and right-sided 


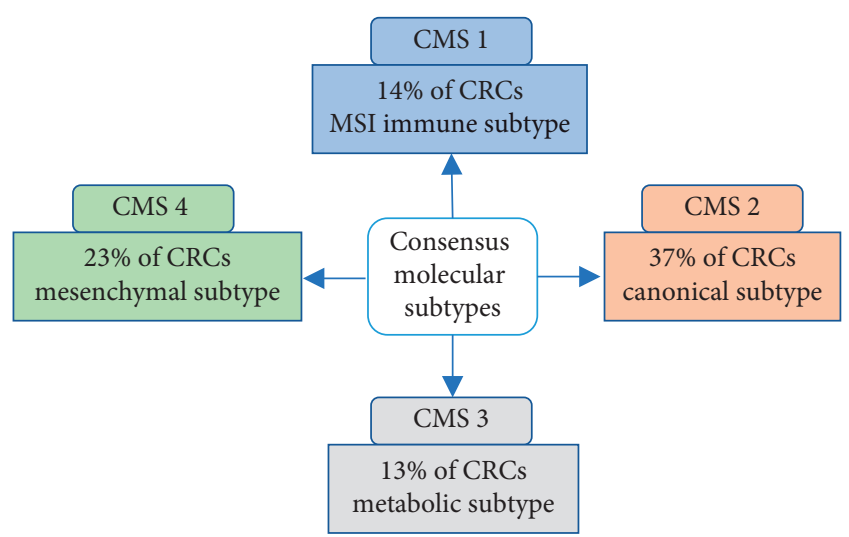

FIGURE 2: Consensus molecular subtypes classification of colorectal cancer. CMS: Consensus Molecular Subtype; CRCs: colorectal cancers; MSI: microsatellite instability.

lesions, conversely to CMS2 tumors which are more frequently left-sided. CMS4 is diagnosed often at advanced stages compared with other subtypes [50].

By analyzing data from patients enrolled in the PETACC-3 clinical trial [58], Guinney et al. [50] concluded that CMS can be used as a prognostic factor [59], and this was also supported by other studies [60-65]. However, as no targeted therapy regimens are available for primary CRC, stratifying tumors using CMS as a prognostic tool needs further evaluation [66]. In a recent large monocenter cohort study including 308 CRC tumors, Purcell et al. concluded that although CMS alone does not surpass TNM staging in terms of prognostication, their combination seems of interest [66]. CMS3 tumors showed lower OS of stage 2 CRCs than other subtypes, whereas stage 2 patients with a good prognosis exhibited immune activation and upregulation of tumor suppressor genes [66].

After analyzing data from the PETACC-3 clinical trial, CMS4 is reported to have the worst OS and relapse-free survival (RFS) in response to fluorouracil (FU)/leucovorin (LV) and irinotecan adjuvant regimen. CMS1 tumors have also a very poor survival rate after relapse, conversely to CMS2 that tends to have superior survival rates after relapse [50].

An in vitro study by Sveen et al. reported a strong response to EGFR and human epidermal growth factor receptor 2 (HER2) inhibitors for the CMS2 group, while CMS1 and CMS4 demonstrated higher sensitivity to HSP90 inhibitors [67]. CMS1 has shown worse PFS and OS in response to anti-EGFR therapy, whereas CMS2 showed particularly better PFS and OS compared with other groups [68]. This was supported by analyzing data from CALGB/SWOG 80405 phase III trial, which found more OS benefit after anti-VEGF than anti-EGFR treatments for the CMS1 group $(P<0.001)$ [60]. However, patients in the CMS2 group treated with cetuximab were found to have better OS compared to those treated with bevacizumab $(P=0.0046)[60]$. Additionally, analysis of data from the FIRE-3 trial showed significantly better OS for CMS4 group after cetuximab plus FOLFIRI treatment compared to bevacizumab plus FOLFIRI in wild-type RAS mCRC [61].

\section{Targeted Therapy in Metastatic CRC}

5-fluorouracil (5-FU) was the first chemotherapy regimen used for the treatment of advanced CRC. After many failed combination regimens to improve its response rate, leucovorin has shown for the first time an advantage over 5-FU alone in terms of tumor response rate in 1992 [69]. Thereafter and over the past two decades, other drugs have shown more improvement in terms of survival, either with 5-FU or alone, such as irinotecan, capecitabine, and oxaliplatin [70]. With the understanding of molecular pathways in CRC, a number of targeted biologic therapies have been approved by the FDA. The first ones were the monoclonal antibodies (mAbs) targeting VEGF (bevacizumab) and the EGFR (cetuximab and panitumumab). The advancement in chemotherapy and targeted drugs has led to significantly improve overall survival to over 40 months for mCRC patients [8].

4.1. Antiangiogenic Inhibitors Targeting VEGF or Its Receptors. Angiogenesis is a mechanism that allows the creation of new blood vessels from preexisting ones to supply cancerous cells. It plays a crucial role in tumor initiation, growth, and metastasis [71]. For a long time, targeting the angiogenic pathway has been considered an important approach for cancer therapy. Although more than 40 molecules have been found to play an important role in blood vessel recruitment, most studies have focused on VEGF and its receptors [70]. VEGF signaling pathway is a key contributor in the process of angiogenesis, and high levels of VEGF ligands and its receptors activity were shown to be related to poor prognosis in CRC and other cancers [72-74].

Bevacizumab is the first anti-VEGF drug that has been approved by the FDA in 2004 for the treatment of patients with $\mathrm{mCRC}$, initiating its use as standard first-line treatment in combination with chemotherapy. Bevacizumab is a humanized monoclonal antibody that binds to VEGF-A, preventing its binding on its receptors. According to a phase II clinical trial of Kabbinavar et al., bevacizumab with FU and LV, in the first line of mCRC treatment, was found to improve significantly the progression-free survival (PFS) and response rate (RR) compared to FU/LV plus placebo (PFS: 9 vs. 5.2 months; hazard ratio (HR), 0.005; $P<0.001$; RR: $40 \%$ vs. $17 \% ; P=0.029)$ but did not improve the median OS (21.5 vs. 13.8 months; $P=0.137$ ). The optimal dose of bevacizumab was $5 \mathrm{mg} / \mathrm{kg}$ [75]. In a phase III trial, significant improvement in OS was demonstrated with irinotecan, FU and LV (IFL), and bevacizumab compared to IFL plus placebo (20.3 vs. 15.6 months; HR, 0.66; $P<0.001)$ [76]. Other clinical trials have tested new combinations with bevacizumab in $\mathrm{mCRC}$ and showed a significant improvement in OS or PFS in first-line setting such as LV calcium, FU, and oxaliplatin-4/capecitabine and oxaliplatin (FOLFOX-4/Xelox), FU, LV, and irinotecan (FOLFIRI) and capecitabine [77-79]. Bevacizumab with FOLFIRI showed a better PFS and OS compared to bolus FU/LV with irinotecan (mIFL) and oral capecitabine with irinotecan (capeIRI) [80]. In the second-line setting, bevacizumab was tested with 
FOLFOX-4 and was shown to improve the median PFS and the RR compared to FOLFOX-4 alone (PFS: 7.3 vs. 4.7 months; HR, 0.61; $P \leq 0.0001 ; \quad R R: 22.7 \%$ vs. $8.6 \%$; $P \leq 0.0001)$ [81]. The ML18147 phase III clinical trial concluded that the continuation of bevacizumab with switching second-line-based chemotherapy improved significantly OS compared to chemotherapy alone or bevacizumab alone [82]. Based on the positive results of these studies, the use of bevacizumab with fluoropyrimidine-oxaliplatin or fluoropyrimidine-irinotecan chemotherapy has been approved by the FDA as a second line for mCRC in 2013.

After bevacizumab, three other antiangiogenic agents have been approved for mCRC: aflibercept, regorafenib, and ramucirumab.

Aflibercept (also known as VEGF Trap, AVE0005) is a recombinant fusion protein that acts as a receptor, binding to human VEGF-A, VEGF-B, and placental growth factor (PGF). It is composed of extracellular domains of human VEGF receptors 1 and 2, fused to the Fc portion of human immunoglobulin (Ig)G1. Aflibercept has a high-affinity ligand trap and prevents these ligands to bind to their endogenous receptors [83]. VELOUR phase III trial showed that treatment with FOLFIRI and aflibercept conferred a significant benefit in terms of OS and PFS compared with FOLFIRI and placebo (OS: 13.5 vs. 12.1 months; $H R, 0.82$; $P=0.0032$; PFS: 6.9 vs. 4.7 months; HR, $0.76 ; P<0.001$ ) [84]. Based on the positive results of this clinical trial, aflibercept has been approved after oxaliplatin failure as a second-line treatment in combination with FOLFIRI in mCRC patients by the FDA in August 2012 and by the European Medicines Agency (EMA) in February 2013.

Regorafenib is an oral multikinase inhibitor that inhibits angiogenic tyrosine kinases (vascular endothelial growth factor receptor 1 to 3 (VEGFR1-3), platelet-derived growth factor receptor (PDGFR- $\beta$ ), and fibroblast growth factor receptor (FGFR1)). It also blocks BRAF and oncogenic receptor tyrosine kinases (RTKs), such as RET and KIT [85]. The CORRECT phase III trial showed that treatment with regorafenib conferred a significant improvement in OS compared with the placebo arm, for mCRC that was refractory to standard therapy (6.4 vs. 5 months; HR, 0.77; $P=0.0052$ ) [86]. Based on this trial, regorafenib has been approved by the FDA in September 2012 for the treatment of mCRC patients who have been treated previously with fluoropyrimidine-, oxaliplatin- and irinotecan-based chemotherapy, an anti-VEGF therapy, and an anti-EGFR therapy if KRAS wild type.

Ramucirumab is a monoclonal human antibody that has a high affinity to VEGFR-2, the essential receptor of the VEGF angiogenic signaling pathway. The RAISE phase III clinical trial has found that ramucirumab plus FOLFIRI as second-line treatment of mCRC improved significantly OS compared with FOLFIRI plus placebo (13.1 vs. 11.7 months, $\mathrm{HR}, 0.84 ; P=0.0219$ ) [87]. Based on these results, ramucirumab (Cyramza) in combination with FOLFIRI has been approved by the FDA as a second-line option for mCRC on April 24, 2015.

Other small multitargeted receptor tyrosine kinase inhibitors (RTKIs) are under investigation for mCRC treatment, such as Famitinib which targets VEGFR2, PDGFR, and c-Kit [88], as well as Fruquintinib and Cediranib which inhibit VEGF1-3 receptors [89, 90]. The main antiangiogenic RTKIs agents, which are under clinical investigation in mCRC, were summarized in Table 1.

4.2. Anti-EGFR Inhibitors. Epidermal growth factor receptor (EGFR/ERBB1) is a member of the erythroblastosis oncogene $B$ (ERBB) family, which also consists of three other receptors, HER2 (ERBB2), HER3 (ERBB3), and HER4 (ERBB4) [91]. Ligands such as EGF, TGF $\alpha$, Amphiregulin (AREG), and Epiregulin (EREG) activate EGFR by binding to its extracellular domain, leading to the activation of the tyrosine kinase domain in the cytoplasm, which stimulates two major signal-transduction pathways, RAS/RAF/MEK/ ERK (mitogen-activated protein kinase (MAPK)) pathway and PI3K/AKT pathway. These two intracellular pathways play key roles in cell proliferation, survival, and migration $[92,93]$.

There is great evidence that ERBB family members have an important role in the initiation and survival of several solid cancers. Over the last years, many studies have shown the importance of constitutive activation of the EGFR pathway in cancer cell proliferation, stopping apoptosis, and activating metastasis [94-96]. This activation may occur through receptor overexpression and ligand-dependent and independent mechanisms [93]. After the research of Masui et al. in 1983 that provided evidence on the activity of antiEGFR drugs against epidermoid carcinoma cell growth in vivo [97], many studies and clinical trials focused on two classes of anti-EFGR agents, which are the anti-EGFR monoclonal antibodies (cetuximab and panitumumab) and the small-molecule EGFR tyrosine kinase inhibitors [92].

Cetuximab is an anti-EGFR monoclonal antibody $\mathrm{mAb}$ (recombinant immunoglobulin G1 (IgG1)), which has been approved by the FDA in 2004, for the treatment of mCRC in combination with irinotecan after irinotecan-based refractory chemotherapy, or as a single agent for mCRC patients who are intolerant to irinotecan. This approval was based essentially on an open-label, randomized trial that showed a significant improvement in terms of RR and PFS for cetuximab with irinotecan, compared to cetuximab alone, in patients who were refractory to irinotecan-based chemotherapy (RR: 22.9 vs. $10.8 \%$; $P$ : 0.007; PFS: 4.1 vs. 1.5; $P<0.001$ ) [98]. The CRYSTAL phase III clinical trial showed that cetuximab with FOLFIRI as a first line of treatment increased the RR by $10 \%$ (adjusted odds ratio (OR): 1.40; $P=0.004$ ) and reduced the risk of mCRC tumor progression by $15 \%(\mathrm{HR}, 0.85 ; P=0.048)$ compared to FOLFIRI alone, and this benefit has been limited to KRAS wild-type groups (RR: $59.3 \%$ vs. $43.2 \%$ (OR, 1.9); PFS: 9.9 vs. 8.2; HR, 0.68; $P=0.02)$. No improvement in OS has been reported in this study, similarly to the previously mentioned trial [99]. Consequently, cetuximab in combination with FOLFIRI has been approved by the FDA for KRAS wild-type mCRC patients as a first-line treatment. FOLFOX4 has been tested in phase II of OPUS clinical trial and showed an improvement in overall response rate (ORR) and a lower risk of 
TABLE 1: Antiangiogenic RTKIs agents under clinical trials investigation in mCRC.

\begin{tabular}{|c|c|c|c|c|c|c|c|}
\hline Drugs & Target & $\begin{array}{l}\text { Number of } \\
\text { participants }\end{array}$ & Setting & Treatment & $\begin{array}{l}\text { Primary } \\
\text { outcome } \\
\text { measures }\end{array}$ & Phase & Identifiers \\
\hline \multirow{2}{*}{$\begin{array}{l}\text { Vatalanib } \\
\text { (PTK787/ZK } \\
\text { 222584) }\end{array}$} & VEGFR1-3, & 1168 & 1st line $\mathrm{mCRC}$ & Oxaliplatin/5FU/ & PFS & \multirow[b]{2}{*}{ III } & NCT00056459 \\
\hline & $\begin{array}{l}\text { FGFR1-3, } \\
\text { PDGFR } \alpha-\beta\end{array}$ & 855 & 2nd line mCRC & Leucovorin \pm vatalanib & OS & & NCT00056446 \\
\hline \multirow{2}{*}{$\begin{array}{l}\text { Nintedanib } \\
\text { (BIBF1120) }\end{array}$} & VEGFR1-3, & 768 & $\begin{array}{l}\text { Refractory } \\
\text { mCRC }\end{array}$ & BSC \pm Nintedanib & PFS/OS & III & NCT02149108 \\
\hline & $\begin{array}{l}\text { FGFR1-3, } \\
\text { PDGFR } \alpha-\beta\end{array}$ & 54 & $\begin{array}{l}\text { Refractory } \\
\text { mCRC }\end{array}$ & mFOLFOX6 \pm Nintedanib & PFS & III & NCT01362361 \\
\hline $\begin{array}{l}\text { Semaxanib } \\
\text { (SU5416) }\end{array}$ & VEGFR2 & 710 & 1st line mCRC & $\begin{array}{l}\text { Leucovorin and } \\
\text { Fluorouracil } \pm \text { Semaxanib }\end{array}$ & OS & III & NCT00004252 \\
\hline Brivanib & $\begin{array}{l}\text { VEGFR2/ } \\
\text { FGFR1 }\end{array}$ & 750 & $\begin{array}{l}\text { mCRC kRAS } \\
\text { wild-type } \\
\text { (refractory) }\end{array}$ & Cetuximab \pm Brivanib & OS & III & NCT00640471 \\
\hline Sunitinib & $\begin{array}{l}\text { PDGFR- } \beta \text {, } \\
\text { VEGFR2, }\end{array}$ & 768 & 1st line mCRC & FOLFIRI \pm Sunitinib & PFS & III & NCT00457691 \\
\hline Fruquintinib & VEGFR1-3 & 416 & 3rd line $\mathrm{mCRC}$ & Fruquintinib vs. placebo & OS & III & NCT02314819 \\
\hline $\begin{array}{l}\text { Cediranib } \\
(\text { AZD2171) }\end{array}$ & VEGFR1-3 & 1814 & 1st line $\mathrm{mCRC}$ & $\begin{array}{l}\text { Beva }+ \text { folfox vs. } \\
\text { beva }+ \text { Cediranib }\end{array}$ & PFS & II/III & NCT00384176 \\
\hline Sorafenib & $\begin{array}{l}\text { VEGFR1-3 } \\
\text { PDGFR- } \beta \\
\text { BRAF }\end{array}$ & 101 & 2nd line mCRC & $\begin{array}{c}\text { FOLFOX6/ } \\
\text { FOLFIRI } \pm \text { Sorafenib }\end{array}$ & PFS & II & NCT00889343 \\
\hline Vanucizumab & $\begin{array}{c}\text { VEGF-A } \\
\text { Angiopoietin-2 }\end{array}$ & 197 & 1st line mCRC & $\begin{array}{c}\text { mFOLFOX6 + beva vs. } \\
\text { mFOLFOX6 + Vancizumab }\end{array}$ & PFS & II & NCT02141295 \\
\hline Famitinib & $\begin{array}{l}\text { VEGFR2-3, c- } \\
\text { Kit, PDGFR }\end{array}$ & 154 & $\begin{array}{c}\text { 3rd line } \\
\text { advanced mCRC }\end{array}$ & Famitinib vs. placebo & PFS & II & NCT01762293 \\
\hline Axitinib & VEGFR1-3 & 70 & $\begin{array}{c}\text { 1st line } \\
\text { maintenance } \\
\text { therapy }\end{array}$ & $\begin{array}{l}\text { Axitinib alone after FOLFOX/ } \\
\text { beva in 1st line }\end{array}$ & PFS & II & NCT01490866 \\
\hline Apatinib & VEGFR2 & 54 & $\begin{array}{l}\text { Refractory } \\
\text { mCRC } \\
\text { progressed after } \\
\text { 2nd line }\end{array}$ & Apatinib alone & PFS & II & NCT03190616 \\
\hline
\end{tabular}

mCRC: metastatic colorectal cancer; 5FU: 5-fluorouracil; FOLFOX: oxaliplatin in combination with 5-fluorouracil and folinic acid; FOLFIRI: irinotecan in combination with 5-fluorouracil and folinic acid; BSC: best supportive care; Beva: bevacizumab; PFS: progression-free survival; OS: overall survival; VEGFR: vascular endothelial growth factor receptor; FGFR: fibroblast growth factor receptor; PDGFR: platelet-derived growth factor receptor.

disease progression in patients with KRAS wild-type disease treated with cetuximab, as compared to those who received FOLFOX4 alone (ORR = 61 vs. $37 \%$; OR: $2.54 ; P=0.011$; PFS: 7.7 vs. 7.2 months; HR, 0.57; $P=0.0163$, respectively) [100]. A pooled analysis of the CRYSTAL and OPUS randomized clinical trials has shown that combining cetuximab to chemotherapy (FOLFIRI or FOLFOX4) in patients with KRAS wild-type disease improved significantly OS (HR, $0.81 ; P=0.0062)$, PFS (HR, $0.66 ; P<0.0001)$, and ORR (OR, 2.16; $P<0.0001)$, compared with chemotherapy alone. In contrast, BRAF mutation status did not show any significant difference in response to cetuximab, but it was a negative prognostic biomarker [101].

Recently, on April 8, 2020, the FDA approved encorafenib in combination with cetuximab for the treatment of mCRC with a BRAF V600E mutation, after prior therapy. This approval was based on the phase III BEACON CRC study, which showed an improvement of median OS for mCRC patients with BRAF V600E treated with cetuximab and encorafenib compared to cetuximab alone (8.4 vs. 5.4 months; HR, $0.6 ; P<0.001)$ [102]. In the second-line setting, the phase III EPIC study has shown that cetuximab and irinotecan improved significantly PFS and RR and resulted in a better quality of life, after oxaliplatin and fluoropyrimidine failure, compared with irinotecan alone [103]. Additionally, cetuximab alone was shown to improve OS and PFS, in wild-type KRAS patients who failed all other treatments, compared with best supportive care (BSC) alone (9.5 vs. 4.8 months; HR, 0.55; $P<0.001$ and 3.7 vs. 1.9 months; HR, 0.40; $P<0.001$, respectively) [104].

Panitumumab is also an anti-EGFR monoclonal antibody. Conversely to cetuximab, it is a fully human IgG2, which has shown reduced immunogenic reactions and high affinity and specificity for the EGF receptors [105]. In phase III clinical trial, panitumumab and BSC showed a significant improvement in PFS for mCRC patients who had progressed after standard chemotherapy, compared with those receiving BSC alone [106]. PRIME phase III study has shown that panitumumab with FOLFOX4 as the first line of treatment for patients with wild-type KRAS mCRC improves significantly PFS compared with FOLFOX4 alone (10 vs. 8.6 months; HR, 0.8; $P=0.01$ ) [107]. As a second-line 
treatment, panitumumab with FOLFIRI has shown significant improvement in terms of PFS, compared with FOLFIRI alone for mCRC patients without KRAS mutations (5.9 vs. 3.9 months; $\mathrm{HR}, 0.71 ; P=0.004$ ) [108]. In 2006, the FDA approved panitumumab as a single agent for the treatment of mCRC KRAS wild-type after chemotherapy regimens failure, and in 2014, it was approved in combination with FOLFOX for patients with wild-type KRAS mCRC in the first-line setting.

\section{Resistance to Anti-EGFR Therapy}

Generally, resistance to targeted drugs englobes primary (de NOVO or innate) and secondary resistance (acquired resistance) [109]. Patients with primary resistance exhibit gene mutations, allelic loss, or gene overexpression, which inactivate or reduce the effectiveness of the drug targets. As an example, RAS, BRAF, and PIK3CA mutations and loss of PTEN and HER2 overexpression have been involved in the primary resistance to anti-EGFR therapy [110]. It has been shown that $40 \%$ of all mCRC patients will derive benefit from these agents [111]. Hence, identifying predictive biomarkers of response to anti-EGFR mAbs is of utmost importance. With regard to secondary resistance, the underlying mechanisms are the acquired EGFR (S492R) mutation, genetic alterations of RAS, BRAF, HER2, and MET, and the selection effect of preexisting subclones that confer primary resistance to anti-EGFR mAbs. These mechanisms and others have been reviewed by Misale et al. and Zhoa et al. [112, 113].

5.1. Primary Resistance. RAS mutations: the frequency of KRAS gene mutations is estimated at $40 \%$ in all CRCs. These mutations were shown to directly activate the MAP kinase signaling pathway, leading to anti-EGFR mAbs resistance [70]. Mutations located on exon2 (codon 12 or 13) were considered the first and the most important predictive biomarker for the nonresponse to anti-EGFR mAbs. These alterations represent $85-90 \%$ of KRAS mutations in CRC, and approximately $40 \%$ of mCRC patients were found to be mutation carriers $[113,114]$. Based on the results of many studies that reported a nonbenefit from anti-EGFR mAbs and shorter PFS, OS, and RR for patients with KRAS exon2 mutations compared with wild-type patients [104, 115-117], the use of anti-EGFR mAbs has been limited by the FDA in 2009 to KRAS exon 2 wild-type mCRC patients [118]. Moreover, other KRAS mutations in exon 3 (codons 59 and 61) and exon 4 (codons 117 and 146) and mutations of the NRAS isoform (exons 2, 3, and 4) were identified in 15-20\% of KRAS exon 2 wild-type patients and were found to be related to low PFS and OS in patients treated with cetuximab and panitumumab [119-122]. Of note, not all patients with wild-type RAS respond to anti-EGFR treatment; that is why research and identification of other biomarkers are of utmost importance.

BRAF mutations: BRAF is a downstream effector, which is directly regulated by RAS. Mutations in the BRAF gene represent 5-9\% of CRCs and may lead to direct activation of the RAS/RAF/ERK pathway [123]. V600E is considered the most commonly reported mutation in BRAF, accounting for more than $95 \%$ of all identified mutations. This mutation causes direct activation of the MAP kinase pathway, leading to resistance to anti-EGFR mAbs [124]. Many studies reported that BRAF V600E has been associated with poorer PFS and OS in patients treated with anti-EGFR mAbs $[125,126]$. However, a meta-analysis of eight randomized controlled trials by Rowland et al. concluded that there is insufficient evidence to consider BRAF as a predictive biomarker of benefit from anti-EGFR mAbs therapy for RAS wild-type mCRC, as there was no statistically significant difference in OS and PFS between RAS wild-type/BRAF wild-type and RAS wild-type/BRAF mutant [127].

PIK3CA mutations and PTEN loss: PI3K-AKT-mTOR signaling pathway is also known to be activated by EGFR, leading to cell proliferation, cell growth, and apoptosis suppression in CRCs [128]. Mutations on PIK3CA, which represent $10-18 \%$ of CRCs, can lead to direct activation of the PI3K/AKT pathway and cause resistance to anti-EGFR mAbs [113]. Mutations in exons 9 and 20 account for $80 \%$ of all PIK3CA mutations and result in its activation and the activation of its downstream signaling pathway [129]. A study by Sartore-Bianchi et al. including 110 mCRC-treated patients reported that PIK3CA mutations conferred significant clinical resistance to cetuximab and panitumumab [130]. Another large retrospective consortium analysis study showed that only PIK3CA exon 20 mutations have been associated with lower RR, PFS, and OS as a response to cetuximab plus chemotherapy compared with wild types, whereas exon 9 mutations showed no effect [131]. A metaanalysis by Mao et al., which included 576 mCRC patients, found that the objective response rate to anti-EGFR mAbs in the KRAS wild-type group was lower in patients with PIK3CA exon 20 mutations, but this difference was not significant due to the limited sample size. However, this study suggested that PIK3CA exon 20 mutations may predict the resistance to anti-EGFR mAbs in KRAS wild-type mCRC patients [132].

PTEN is considered a suppressor gene, due to its role in the negative regulation of AKT. Therefore, loss of PTEN expression or function leads to persistent activation of the PI3K-AKT-mTOR signaling pathway, which results in permanent cell proliferation and growth [133]. Some studies have found that PTEN can be a useful predictive biomarker for the response to anti-EGFR mAbs therapy, particularly in the KRAS wild-type [134-137]. In contrast, other studies did not find a significant difference in response to anti-EGFR therapy between PTEN-positive and PTEN-negative groups [138-140]. To confirm the role of PTEN in anti-EGFR resistance, other large clinical studies are warranted.

Level of EGFR ligand expression: in addition to studying the downstream EGFR signaling pathway in antiEGFR resistance, the upregulation has also been investigated in some studies, especially in an intact downstream EGFR pathway [141-144]. In an exploratory cetuximab monotherapy clinical trial, cetuximab efficacy has been found to be related to KRAS wild-type and high gene expression levels of AREG and EREG in mCRC patients 
[145], whereas other clinical trials confirmed that AREG and EREG expression levels have predictive power to antiEGFR therapy only for KRAS wild-type patients $[142,146]$. A recent study that analyzed tumor tissue of 688 patients participating in FIRE-1, CIOX, and FIRE-3 clinical trials has confirmed that AREG high level is a positive prognostic biomarker for anti-EGFR therapy in mCRC. AREG high level was significantly associated with high OS and PFS compared to a low level (26.2 vs. 21.5 months; $P=0.007)$ (10.0 vs. 8.1 months; $P=0.001$ ), respectively [141]. EGFR ligand's high expression level can be related to tumors' dependence on the EGFR signaling pathway, which explains its predictive power in mCRC, but it can also occur as a consequence of epigenetic regulation [147].

STAT3: STAT3 is a transcription factor belonging to the STAT family. It is an essential component of the JAK/STAT signaling pathway, which is activated by EGFR and other receptors [148]. Phosphorylated by JAK, STAT3 plays an important role in cell proliferation, survival, and apoptosis activities $[113,149]$. Persistent STAT3 activation may have a key role in anti-EGFR therapy resistance in mCRC. A retrospective study by Dobi et al. reported a significant improvement in time to progression (TTP) and OS for negative phospho-STAT3 compared to positive phospho-STAT3 group (TTP: 6.3 vs. 5.4 months; $P<0.01$; OS: 13.1 vs. 9.4 months; $P=0.02$ ), among $94 \mathrm{mCRC}$ patients who were treated with cetuximab and chemotherapy in the second-line setting or beyond [150]. Another study by Ung et al. indicated a key role of STAT3 in promoting resistance to antiEGFR treatment and showed that STAT3 activity can be inhibited by the anti-EGFR inhibitors in wild-type K-RAS colon cancer cell lines, suggesting that anti-EGFR therapy combined with STAT3 inhibitors may provide a therapeutic benefit for mCRC patients [151].

5.2. Secondary Resistance. Acquisition of KRAS mutation: as we mentioned previously, the RAS/RAF signaling pathway plays an important role in primary resistance to anti-EGFR $\mathrm{mAbs}$ in mCRC, but it has also been involved in acquired resistance [152]. A report by Diaz et al. found that $38 \%$ of KRAS wild-type patients who received panitumumab had KRAS mutations in their sera, occurring after five or six months of the treatment [153]. Interestingly, a mathematical model from this study showed that resistance mutations in KRAS were present in a clonal subpopulation within the tumors before initiating panitumumab therapy [153]. Another study reported that $55 \%(6 / 11)$ of patients who developed cetuximab or panitumumab resistance harbored secondary k-RAS mutations, and 9\% (1/11) had K-RAS amplification [154]. KRAS variants were detectable in plasma of cetuximab-treated patients 10 months before radiographic documentation of disease progression [154].

EGFR S492R mutation: Montagut et al. were the first to report in 2012 that EGFR S492R mutation confers acquired resistance in $\mathrm{mCRC}$ patients treated with cetuximab, but not in those who were treated with panitumumab. In their study, $20 \%$ of patients who showed resistance to cetuximab harbored EGFR S492R mutation [155]. Later, a larger cohort study including 505 mCRC KRAS exon 2 wild-type patients suggested that EGFR S492R mutation was not involved in primary resistance to cetuximab [156]. In the ASPECCT study, a randomized controlled phase III trial, EGFR S492R mutation was detected, after analyzing liquid biopsies, in $16 \%$ of patients treated with cetuximab compared to $1 \%$ in those receiving panitumumab [157].

Amplification of HER2: HER2 can activate the RAS/ RAF/ERK and PI3K/AKT pathways through its heterodimerization with EGFR and HER3, which are all members of the ERBB receptors tyrosine kinase family. For this reason, HER2 is considered a potential biomarker for the sensitivity to anti-EGFR therapy [91]. Studies using patientderived xenografts showed that HER2 amplification or the overexpression of heregulin ligand leads to cetuximab resistance especially in KRAS and BRAF wild-type cancers $[158,159]$. These findings have been supported by clinical data from a large retrospective cohort study by Martin et al. showing that HER2 gene copy number may confer resistance to anti-EGFR therapy in KRAS wild mCRC type [160]. HER2 amplification may also be involved in primary resistance, but considering its low frequency ( $2 \%$ of mCRC patients), it is mainly considered a mechanism of secondary resistance to anti-EGFR [113].

MET amplification: mesenchymal-epithelial transition factor (c-MET) is a tyrosine kinase receptor, which is encoded by the protooncogene MET. Binding to its ligand the hepatocyte growth factor (HGF), c-MET induces cell proliferation, growth, survival, and angiogenesis, through the activation of PI3K/AKT, RAS/RAF/ERK, STAT3, and nuclear factor $-\kappa \mathrm{B}(\mathrm{NF}-\kappa \mathrm{B})$ signaling pathways [161]. Liska et al. have confirmed the importance of MET activation in restoring the MAPK and AKT pathways during anti-EGFR therapy in CRC cell lines [162]. In both in vitro and in vivo settings, Bardelli et al. demonstrated that MET amplification confers acquired resistance in patients who were KRAS wildtype during anti-EGFR therapy. Importantly, findings from this study supported using blood tests to monitor the emergence of MET amplification in patients undergoing anti-EGFR therapies, as the amplification of the MET locus has been present in circulating tumor DNA before any clinical evidence of relapse [163]. Another study by Troiani et al. showed that TGF- $\alpha$ overexpression induces the EGFRMET interaction leading to subsequent MET pathway activation and MET acquired resistance and suggested that the inhibition of MET expression restores the sensitivity to cetuximab in CRC cell lines [164]. Nonetheless, MET amplification occurs only in $1 \%$ of unselected mCRC patients $[163,165]$, making it a weak predictive biomarker of primary resistance to anti-EGFR therapy in MCRC [113].

The subclone selection: the notion of secondary resistance not only consists of the development of new mutations during the therapy but can also include the selection of a low-frequency subclone that conferred primary resistance under the target therapy pressure [166]. To determine whether the acquired resistance to cetuximab in mCRC patients is due to novel mutations or the selection of preexisting subclones, Misale et al. compared gene copy number and the mutational profile of parental and resistant cell lines 
TABLE 2: mAbs targeting EGFR pathway under clinical investigation.

\begin{tabular}{|c|c|c|c|c|c|}
\hline Drugs & Target & Setting & Treatment & Phase & Identifiers \\
\hline Trastuzumab & HER2 & $\begin{array}{l}\text { HER2-positive wild KRAS } \\
\text { mCRC }\end{array}$ & $\begin{array}{c}\text { Trastuzumab + Lapatinib or Pertuzumab } \\
\text { Trastuzumab + Tucatinib }\end{array}$ & II & $\begin{array}{l}\text { NCT03225937 } \\
\text { NCT03043313 }\end{array}$ \\
\hline Pertuzumab & HER2 & 2nd line of advanced or mCRC & Pertuzumab + cetuximab & $\mathrm{I} / \mathrm{II}$ & NCT00551421 \\
\hline $\begin{array}{l}\text { MEHD7945A } \\
\text { (Duligotuzumab) }\end{array}$ & $\begin{array}{l}\text { EGFR/ } \\
\text { HER3 }\end{array}$ & 2nd line K-Ras wild-type mCRC & $\begin{array}{l}\text { MEHD7945A + FOLFIRI vs. } \\
\text { Cetuximab + FOLFIRI }\end{array}$ & II & NCT01652482 \\
\hline SYM004 & EGFR & $\begin{array}{l}\text { mCRC K-Ras WT acquired } \\
\text { resistance to Anti-EGFR mAbs }\end{array}$ & SYM004 vs. BSC & II & NCT02083653 \\
\hline CPGJ 602 & EGFR & 2nd line mCRC KRAS WT & CPGJ 602 vs. Cetuximab & I & NCT03356158 \\
\hline Futuximab & EGFR & $\begin{array}{c}\text { Chemotherapy-refractory } \\
\text { mCRC }\end{array}$ & Futuximab vs. SYM004 & II & NCT03549338 \\
\hline SCT 200 & EGFR & Wild-type RAS and RAF mCRC & SCT200 & II & NCT03405272 \\
\hline $\begin{array}{l}\text { Dalotuzumab (MK- } \\
\text { 0646) }\end{array}$ & IGF-1R & Wild-type KRAS mCRC & Dalotuzumab + Cetuximab + irinotecan & II/III & NCT00614393 \\
\hline $\begin{array}{l}\text { Ganitumab (AMG- } \\
479)\end{array}$ & IGF-1R & Mutant KRAS mCRC & AMG-479 + FOLFIRI vs. FOLFIRI alone & II & NCT00813605 \\
\hline $\begin{array}{l}\text { Cixutumumab (IMC- } \\
\text { A12) }\end{array}$ & IGF-1R & 2nd line mCRC kRAS wild-type & Irinotecan and Cetuximab \pm IMC-A12 & II & NCT00845039 \\
\hline
\end{tabular}

TABLE 3: TKIs targeting EGFR pathway under clinical investigation.

\begin{tabular}{|c|c|c|c|c|c|}
\hline Drugs & Target & Setting & Treatment & Phase & Identifiers \\
\hline Erlotinib & EGFR & 2nd line k-RAS WT mCRC & Erlotinib + panitumumab \pm irinotecan & II & NCT00940316 \\
\hline Neratinib & EGFR/HER2 & $\begin{array}{l}\text { KRAS/NRAS/BRAF/ } \\
\text { PIK3CA wild-type mCRC }\end{array}$ & $\begin{array}{l}\text { Neratinib + Trastuzumb vs. } \\
\text { Neratinib + Cetuximab }\end{array}$ & II & NCT03457896 \\
\hline $\begin{array}{l}\text { Sapitinib } \\
\text { (AZD8931) }\end{array}$ & $\begin{array}{l}\text { EGFR/ } \\
\text { HER2/3 }\end{array}$ & $\begin{array}{c}\text { Recurrent or metastatic } \\
\text { CRC }\end{array}$ & AZD8931 + FOLFIRI & II & NCT01862003 \\
\hline Tucatinib & HER2 & HER2 positive CRC & Tucatinib + trastuzumab & II & NCT03043313 \\
\hline $\begin{array}{l}\text { Lapatinib } \\
(\text { GSK572016) }\end{array}$ & $\begin{array}{c}\text { EGFR/ } \\
\text { HER2/erk-1/ } \\
2\end{array}$ & $\begin{array}{l}\text { 2nd line advanced or } \\
\text { mCRC }\end{array}$ & Lapatinib + capecitabine & II & NCT00574171 \\
\hline Vemurafenib & $\begin{array}{c}\text { BRAF } \\
(\mathrm{V} 600 \mathrm{E})\end{array}$ & $\begin{array}{l}\text { BRAF V600E mutation and } \\
\text { advanced CRC }\end{array}$ & FOLFIRI + Cetuximab + Vemurafenib & II & NCT03727763 \\
\hline Dabrafenib & $\begin{array}{l}\text { BRAF } \\
(\mathrm{V} 600 \mathrm{E})\end{array}$ & $\begin{array}{l}\text { BRAF V600E mutation } \\
\text { mCRC }\end{array}$ & Dabrafenib + trametinib + spartalizumab & II & NCT03668431 \\
\hline $\begin{array}{l}\text { Encorafenib (LGX- } \\
818)\end{array}$ & $\begin{array}{l}\text { BRAF } \\
(\mathrm{V} 600 \mathrm{E})\end{array}$ & MSS/BRAF V600E mCRC & Encorafenib + Cetuximab + nivolumab & $\mathrm{I} / \mathrm{II}$ & NCT04017650 \\
\hline BMS-908662 & BRAF & Mutant BRAF mCRC & BMS-908662 + cetuximab & $\mathrm{I} / \mathrm{II}$ & NCT01086267 \\
\hline $\begin{array}{l}\text { Binimetinib } \\
\text { (MEK162) }\end{array}$ & MEK $1 / 2$ & RAS positive mCRC & Binimetinib + mFOLFIRI & I & NCT02613650 \\
\hline Cobimetinib & MEK1 & $\begin{array}{l}\text { Locally advanced and } \\
\text { metastatic CRC }\end{array}$ & Cobimetinib + Atezolizumab vs. Regorafenib & III & NCT02788279 \\
\hline Trametinib & $\mathrm{MEK} 1 / 2$ & $\begin{array}{l}\text { RAS/RAF mutant and TP53 } \\
\text { WT mCRC }\end{array}$ & Trametinib + HDM201 & I & NCT03714958 \\
\hline $\begin{array}{l}\text { Selumetinib } \\
\text { (AZD6244) }\end{array}$ & MEK1/2 & $\begin{array}{c}\text { 2nd line k-RAS BRAF } \\
\text { mCRC }\end{array}$ & Selumetinib + irinotecan & II & NCT01116271 \\
\hline Alpelisib (BYL719) & PI3K & BRAF mutant mCRC & $\begin{array}{c}\text { Alpelisib + Cetuximab vs. } \\
\text { BYL719 + Cetuximab + LGX818 }\end{array}$ & $\mathrm{I} / \mathrm{II}$ & NCT01719380 \\
\hline $\begin{array}{l}\text { Buparlisib } \\
\text { (BKM120) }\end{array}$ & PI3K & $\begin{array}{l}\text { Wild-type RAS advanced or } \\
\text { metastatic CRC }\end{array}$ & Panitumumab + BKM120 & $\mathrm{I} / \mathrm{II}$ & NCT01591421 \\
\hline $\begin{array}{l}\text { Gedatolisib } \\
\text { (PF05212384) }\end{array}$ & $\mathrm{PI} 3 \mathrm{~K} / \mathrm{mTOR}$ & mCRC & $\begin{array}{l}\text { Gedatolisib + FOLFIRI vs. } \\
\text { FOLFIRI + Bevacizumab }\end{array}$ & $\mathrm{I} / \mathrm{II}$ & NCT01937715 \\
\hline $\begin{array}{l}\text { Nab-rapamycin } \\
\text { (ABI-009) }\end{array}$ & mTOR & $\begin{array}{l}\text { 1st line advanced or } \\
\text { metastatic CRC }\end{array}$ & ABI-009 + FOLFOX + bevacizumab & $\mathrm{I} / \mathrm{II}$ & NCT03439462 \\
\hline $\begin{array}{l}\text { Everolimus } \\
\text { (RAD001) }\end{array}$ & mTOR & 2nd line $\mathrm{mCRC}$ & Irinotecan + Cetuximab \pm Everolimus & $\mathrm{I} / \mathrm{II}$ & NCT00522665 \\
\hline
\end{tabular}


TABLE 3: Continued.

\begin{tabular}{|c|c|c|c|c|c|}
\hline Drugs & Target & Setting & Treatment & Phase & Identifiers \\
\hline ONC201 & AKT/ERK & MSS mCRC & ONC201 + Nivolumab & I/II & NCT03791398 \\
\hline MK2206 & AKT & $\begin{array}{l}\text { WT k-RAS/mutated } \\
\text { PIK3CA mCRC }\end{array}$ & MK2206 & II & NCT01186705 \\
\hline TTI-101 & STAT3 & Advanced CRC & TTI-101 & I & NCT03195699 \\
\hline Ruxolitinib & JAK/STAT3 & $\begin{array}{l}\text { RAS mutant advanced CRC } \\
\text { and pancreatic cancer }\end{array}$ & Ruxolitinib + trametinib & $\mathrm{I}$ & NCT04303403 \\
\hline
\end{tabular}

mCRC: metastatic colorectal cancer; FOLFIRI: irinotecan in combination with 5-fluorouracil and folinic acid; MSS: microsatellite stability; FOLFOX: leucovorin calcium, fluorouracil, and oxaliplatin; KRAS: Kirsten rat sarcoma viral oncogene; WT Kras: wild-type Kras; HER: human epidermal growth factor receptor; EGFR: epidermal growth factor receptor; BRAF: v-raf murine sarcoma viral oncogene homolog B1; PIK3: phosphatidylinositol 3-kinase; mTOR: mammalian target of rapamycin. JAK: Janus kinase; STAT3: signal transducer and activator of transcription 3.

[154]. This study found that KRAS G13D mutation and KRAS amplification were present in parental cells with low frequency, which supports the theory of subclonal selection [154]. However, other mutations including KRAS G12R and EGFR S492R have been identified only in resistant cells $[154,156]$, which corroborates that some mutations may occur only during the EGFR mAbs treatment.

5.3. Overcoming Resistance to Anti-EGFR Therapy. In order to overcome resistance to cetuximab/panitumumab in mCRC patients, research studies have been focusing on widening therapeutic choices by testing new monoclonal antibodies against EGFR receptors (Duligotuzumab, Futuximab, etc.), as well as those against HER2, HER3, and IGF-1R receptors (Table 2). Using tyrosine kinase inhibitors to target the EGFR receptor and its downstream pathway is another promising active research area (Table 3 ).

\section{Conclusion}

The advancements in molecular technologies in recent decades, especially sequencing techniques, have led to a better understanding of the genomic landscape of CRC and hence increased dramatically our knowledge about the carcinogenesis process. This unprecedented information has been directly harnessed to establish molecular classifications that provide deeper insights into the biology of CRC. Available evidence suggests that the CMS classification might have a predictive and prognostic value, and also it can help to guide drug development and application.

The molecular understanding of CRC carcinogenesis has also led to developing targeted drugs such as anti-VEGF mAbs, anti-EGFR mAbs, and multikinase inhibitors, which improved the survival rates of mCRC patients. However, emerging primary or secondary resistance to current targeted therapies, especially to anti-EGFR mAbs, remains a major problem in clinical practice. Understanding resistance mechanisms, identifying new biomarkers and other targetable pathways is of paramount importance to optimize therapeutic choices and improve survival for resistant patients.

\section{Abbreviations}

APC: Adenomatous polyposis coli

AREG: Amphiregulin
BSC: $\quad$ Best supportive care

CapeIRI: Capecitabine and irinotecan

CIMP: $\quad$ CpG Island Methylator Phenotype

CIN: Chromosomal instability

c-MET: Mesenchymal epithelial transition factor

CMS: $\quad$ Consensus molecular subtype

CRC: $\quad$ Colorectal cancer

dMMR: Deficient mismatch repair

EGFR: Epidermal growth factor receptor

EMA: $\quad$ European medicines agency

EMT: Epithelial-to-mesenchymal transition

ERBB: Erythroblastosis oncogene B

EREG: Epiregulin

FAP: $\quad$ Familial adenomatous polyposis

FDA: $\quad$ Food drug administration

FGFR: Fibroblast growth factor receptor

FOLFIRI: Fluorouracil, leucovorin and irinotecan

FOLFOX: Leucovorin calcium, fluorouracil, and oxaliplatin

FU: $\quad$ Fluorouracil

HER: Human epidermal growth factor receptor

HGF: Hepatocyte growth factor

HNPCC: Hereditary nonpolyposis colorectal cancer

HR: $\quad$ Hazard ratio

IDLs: Insertion-deletion loops

IFL: Irinotecan, fluorouracil and leucovorin

IGF-1R: Insulin-like growth factor 1 receptor

IgG: Immunoglobulin G

IHC: Immunohistochemistry

JPS: Juvenile polyposis syndrome

KRAS: Kirsten rat sarcoma viral oncogene

LOH: Loss of heterozygosity

LV: $\quad$ Leucovorin

mAbs: Monoclonal antibodies

MAP: $\quad$ MYH-associated polyposis

MAPK: Mitogen-activated protein kinase

mCRC: Metastatic CRC

MMR: $\quad$ Mismatch repair

MSI: $\quad$ Microsatellite instability

MSI-H: Microsatellite instability high

OR: $\quad$ Odds ratio

ORR: Overall response rate

OS: $\quad$ Overall survival

PDGFR: Platelet-derived growth factor receptor

PFS: $\quad$ Progression-free survival

PGF: Placental growth factor 


$\begin{array}{ll}\text { PJS: } & \text { Peutz Jeghers } \\ \text { POLE: } & \text { Polymerase- } \varepsilon \\ \text { RFS: } & \text { Relapse-free survival } \\ \text { RR: } & \text { Response rate } \\ \text { RTKs: } & \text { Receptor tyrosine kinases } \\ \text { SCNAs: } & \text { Somatic copy number alterations } \\ \text { TCGA: } & \text { The cancer genome atlasTGF: } \\ \text { TGF: } & \text { Transforming growth factor } \\ \text { TTP: } & \text { Time to progression } \\ \text { VEGF: } & \text { Vascular endothelial growth factor } \\ \text { VEGFR: } & \text { Vascular endothelial growth factor receptor } \\ \text { XELOX: } & \text { Capecitabine (xeloda) and oxaliplatin. }\end{array}$

\section{Conflicts of Interest}

The authors declare that they have no conflicts of interest.

\section{Authors' Contributions}

Mouade El Bali was responsible for the conception and design of the study, analyzing literature data, and writing the manuscript. Joaira Bakkach drafted some parts of the work, participated in the design and the conception of the study, and revised it critically. Mohcine Bennani Mechita revised the manuscript. All authors read and approved the final version.

\section{References}

[1] N. Keum and E. Giovannucci, "Global burden of colorectal cancer: emerging trends, risk factors and prevention strategies," Nature Reviews Gastroenterology \& Hepatology, vol. 16, no. 12, pp. 713-732, 2019.

[2] F. Bray, J. Ferlay, I. Soerjomataram, R. L. Siegel, L. A. Torre, and A. Jemal, "Global cancer statistics 2018: GLOBOCAN estimates of incidence and mortality worldwide for 36 cancers in 185 countries," CA: A Cancer Journal for Clinicians, vol. 68, no. 6, pp. 394-424, 2018.

[3] E. Dekker, P. J. Tanis, J. L. A. Vleugels, P. M. Kasi, and M. B. Wallace, "Colorectal cancer," The Lancet, vol. 394, no. 10207, pp. 1467-1480, 2019.

[4] Y.-H. Xie, Y.-X. Chen, and J.-Y. Fang, "Comprehensive review of targeted therapy for colorectal cancer," Signal Transduction and Targeted Therapy, vol. 5, no. 1, p. 22, 2020.

[5] R. L. Siegel, K. D. Miller, and A. Jemal, "Cancer statistics, 2019," CA: A Cancer Journal for Clinicians, vol. 69, no. 1, pp. 7-34, 2019.

[6] P. Rawla, T. Sunkara, and A. Barsouk, "Epidemiology of colorectal cancer: incidence, mortality, survival, and risk factors," Gastroenterology Review, vol. 14, no. 2, pp. 89-103, 2019.

[7] D. Colussi, G. Brandi, F. Bazzoli, and L. Ricciardiello, "Molecular pathways involved in colorectal cancer: implications for disease behavior and prevention," International Journal of Molecular Sciences, vol. 14, no. 8, pp. 16365-16385, 2013.

[8] D. M. Graham, V. M. Coyle, R. D. Kennedy, and R. H. Wilson, "Molecular subtypes and personalized therapy in metastatic colorectal cancer," Current Colorectal Cancer Reports, vol. 12, no. 3, pp. 141-150, 2016.
[9] H. Seow, W. K. Yip, and T. Fifis, "Advances in targeted and immunobased therapies for colorectal cancer in the genomic era," OncoTargets and Therapy, vol. 9, p. 1899, 2016.

[10] H. Sawayama, Y. Miyamoto, K. Ogawa, N. Yoshida, and $\mathrm{H}$. Baba, "Investigation of colorectal cancer in accordance with consensus molecular subtype classification," Annals of Gastroenterological Surgery, vol. 4, no. 5, pp. 528-539, 2020.

[11] E. J. Kuipers, W. M. Grady, D. Lieberman et al., "Colorectal cancer," Nature Reviews Disease Primers, vol. 1, no. 1, p. 15065, 2015.

[12] A. Morán, "Differential colorectal carcinogenesis: molecular basis and clinical relevance," World Journal of Gastrointestinal Oncology, vol. 2, no. 3, p. 151, 2010.

[13] D. G. Power, E. Gloglowski, and S. M. Lipkin, "Clinical genetics of hereditary colorectal cancer," Hematology/Oncology Clinics of North America, vol. 24, no. 5, pp. 837-859, 2010.

[14] S. Al-Sohaily, A. Biankin, R. Leong, M. Kohonen-Corish, and J. Warusavitarne, "Molecular pathways in colorectal cancer," Journal of Gastroenterology and Hepatology, vol. 27, no. 9, pp. 1423-1431, 2012.

[15] K. Tamura, M. Kaneda, M. Futagawa et al., "Genetic and genomic basis of the mismatch repair system involved in Lynch syndrome," International Journal of Clinical Oncology, vol. 24, no. 9, pp. 999-1011, 2019.

[16] L. Valle, E. Vilar, S. V. Tavtigian, and E. M. Stoffel, "Genetic predisposition to colorectal cancer: syndromes, genes, classification of genetic variants and implications for precision medicine," The Journal of Pathology, vol. 247, no. 5, pp. 574-588, 2019.

[17] A. S. Aghabozorgi, A. Bahreyni, A. Soleimani et al., "Role of adenomatous polyposis coli (APC) gene mutations in the pathogenesis of colorectal cancer; current status and perspectives," Biochimie, vol. 157, pp. 64-71, 2019.

[18] F. G. Campos, "Colorectal cancer risk in hamartomatous polyposis syndromes," World Journal of Gastrointestinal Surgery, vol. 7, no. 3, p. 25, 2015.

[19] F. Kastrinos and S. Syngal, "Inherited colorectal cancer syndromes," The Cancer Journal, vol. 17, no. 6, pp. 405-415, 2011.

[20] E. R. Fearon and B. Vogelstein, "A genetic model for colorectal tumorigenesis,” Cell, vol. 61, no. 5, pp. 759-767, 1990.

[21] E. A. H. Kheirelseid, N. Miller, and M. J. Kerin, "Molecular biology of colorectal cancer: review of the literature," American Journal of Molecular Biology, vol. 3, no. 2, pp. 72-80, 2013.

[22] D. L. Worthley, "Colorectal carcinogenesis: road maps to cancer," World Journal of Gastroenterology, vol. 13, no. 28, p. 3784, 2007.

[23] M. S. Pino and D. C. Chung, "The chromosomal instability pathway in colon cancer," Gastroenterology, vol. 138, no. 6, pp. 2059-2072, 2010.

[24] W. M. Grady, "Genomic instability and colon cancer," Cancer and Metastasis Reviews, vol. 23, no. 1-2, pp. 11-27, 2004.

[25] S. M. Powell, N. Zilz, Y. Beazer-Barclay et al., "APC mutations occur early during colorectal tumorigenesis," Nature, vol. 359, no. 6392, pp. 235-237, 1992.

[26] A. B. Sparks, P. J. Morin, B. Vogelstein, and K. W. Kinzler, "Mutational analysis of the APC//3-catenin/Tcf pathway in colorectal cancer," Cancer Research, vol. 58, pp. 1130-1134, 1998. 
[27] M. Brink, "K-ras oncogene mutations in sporadic colorectal cancer in the Netherlands cohort study," Carcinogenesis, vol. 24, no. 4, pp. 703-710, 2003.

[28] J.-Y. Wang, Y.-H. Wang, S.-W. Jao et al., "Molecular mechanisms underlying the tumorigenesis of colorectal adenomas: correlation to activated K-ras oncogene," Oncology Reports, vol. 16, no. 6, pp. 1245-1252, 2006.

[29] G. Tarafa, A. Villanueva, L. Farré et al., "DCC and SMAD4 alterations in human colorectal and pancreatic tumor dissemination," Oncogene, vol. 19, no. 4, pp. 546-555, 2000.

[30] K. L. Woodford-Richens, A. J. Rowan, P. Gorman et al., "SMAD4 mutations in colorectal cancer probably occur before chromosomal instability, but after divergence of the microsatellite instability pathway," Proceedings of the National Academy of Sciences, vol. 98, no. 17, pp. 9719-9723, 2001.

[31] A. J. Cole, Y. Zhu, T. Dwight et al., "Comprehensive analyses of somatic TP53 mutation in tumors with variable mutant allele frequency," Scientific Data, vol. 4, no. 1, Article ID 170120, 2017.

[32] A. Leslie, F. A. Carey, N. R. Pratt, and R. J. C. Steele, "The colorectal adenoma-carcinoma sequence," British Journal of Surgery, vol. 89, no. 7, pp. 845-860, 2002.

[33] E. Vilar and S. B. Gruber, "Microsatellite instability in colorectal cancer-the stable evidence," Nature Reviews Clinical Oncology, vol. 7, no. 3, pp. 153-162, 2010.

[34] J. Jiricny, "The multifaceted mismatch-repair system," $\mathrm{Na}$ ture Reviews Molecular Cell Biology, vol. 7, no. 5, pp. 335346, 2006.

[35] M. Hewish, C. J. Lord, S. A. Martin, D. Cunningham, and A. Ashworth, "Mismatch repair deficient colorectal cancer in the era of personalized treatment," Nature Reviews Clinical Oncology, vol. 7, no. 4, pp. 197-208, 2010.

[36] C. Evrard, G. Tachon, V. Randrian, L. Karayan-Tapon, and D. Tougeron, "Microsatellite instability: diagnosis, heterogeneity, discordance, and clinical impact in colorectal cancer," Cancers, vol. 11, no. 10, p. 1567, 2019.

[37] C. R. Boland, S. N. Thibodeau, S. R. Hamilton et al., "A National Cancer Institute workshop on microsatellite instability for cancer detection and familial predisposition: development of international criteria for the determination of microsatellite instability in colorectal cancer," Cancer Research, vol. 58, pp. 5248-5257, 1998.

[38] C. R. Boland and A. Goel, "Microsatellite instability in colorectal cancer," Gastroenterology, vol. 138, no. 6, pp. 2073-2087, 2010.

[39] G. Deng, I. Bell, S. Crawley et al., "BRAF mutation is frequently present in sporadic colorectal cancer with methylated hMLH1, but not in hereditary nonpolyposis colorectal cancer," Clinical Cancer Research, vol. 10, no. 1, pp. 191-195, 2004.

[40] A. McGivern, C. V. A. Wynter, V. L. J. Whitehall et al., "Promoter hypermethylation frequency and BRAF mutations distinguish hereditary non-polyposis colon cancer from sporadic MSI-H colon cancer," Familial Cancer, vol. 3, no. 2, pp. 101-107, 2002.

[41] M. Esteller, "Epigenetics in cancer," New England Journal of Medicine, vol. 358, pp. 1148-1159, 2008.

[42] S. M. Advani, P. Advani, S. M. DeSantis et al., "Clinical, pathological, and molecular characteristics of CpG island methylator phenotype in colorectal cancer: a systematic review and meta-analysis," Translational Oncology, vol. 11, no. 5, pp. 1188-1201, 2018.
[43] M. Toyota, N. Ahuja, M. Ohe-Toyota, J. G. Herman, S. B. Baylin, and J.-P. J. Issa, "CpG island methylator phenotype in colorectal cancer," Proceedings of the National Academy of Sciences of the United States of America, vol. 96, pp. 8681-8686, 1999.

[44] J. J. L. Wong, N. J. Hawkins, and R. L. Ward, "Colorectal cancer: a model for epigenetic tumorigenesis," Gut, vol. 56, no. 1, pp. 140-148, 2007.

[45] D. J. Weisenberger, K. D. Siegmund, and M. Campan, "CpG island methylator phenotype underlies sporadic microsatellite instability and is tightly associated with BRAF mutation in colorectal cancer," Nature Genetics, vol. 38, no. 7, pp. 787-793, 2006.

[46] Y.-Y. Rhee, K.-J. Kim, and G. H. Kang, "CpG island methylator phenotype-high colorectal cancers and their prognostic implications and relationships with the serrated neoplasia pathway," Gut Liver, vol. 11, no. 1, pp. 38-46, 2017.

[47] Y. Tao, B. Kang, D. A. Petkovich et al., "Aging-like spontaneous epigenetic silencing facilitates Wnt activation, stemness, and BrafV600E-induced tumorigenesis," Cancer Cell, vol. 35, no. 2, pp. 315-328, 2019.

[48] M. F. Müller, A. E. K. Ibrahim, and M. J. Arends, "Molecular pathological classification of colorectal cancer," Virchows Archiv, vol. 469, no. 2, pp. 125-134, 2016.

[49] The Cancer Genome Atlas Network, "Comprehensive molecular characterization of human colon and rectal cancer," Nature, vol. 487, no. 7407, pp. 330-337, 2012.

[50] J. Guinney, R. Dienstmann, X. Wang et al., "The consensus molecular subtypes of colorectal cancer," Nature Medicine, vol. 21, no. 11, pp. 1350-1356, 2015.

[51] P. Roepman, A. Schlicker, J. Tabernero et al., "Colorectal cancer intrinsic subtypes predict chemotherapy benefit, deficient mismatch repair and epithelial-to-mesenchymal transition: molecular subtypes in colorectal cancer," International Journal of Cancer, vol. 134, no. 3, pp. 552-562, 2014.

[52] E. Budinska, V. Popovici, S. Tejpar et al., "Gene expression patterns unveil a new level of molecular heterogeneity in colorectal cancer: gene expression heterogeneity in colorectal cancer," Journal of Pathology, vol. 231, no. 1, pp. 63-76, 2013.

[53] A. Schlicker, G. Beran, C. M. Chresta et al., "Subtypes of primary colorectal tumors correlate with response to targeted treatment in colorectal cell lines," BMC Medical Genomics, vol. 5, no. 1, p. 66, 2012

[54] A. Sadanandam, C. A. Lyssiotis, K. Homicsko et al., "A colorectal cancer classification system that associates cellular phenotype and responses to therapy," Nature Medicine, vol. 19, no. 5, pp. 619-625, 2013.

[55] F. D. S. E. Melo, X. Wang, M. Jansen et al., "Poor-prognosis colon cancer is defined by a molecularly distinct subtype and develops from serrated precursor lesions," Nature Medicine, vol. 19, no. 5, pp. 614-618, 2013.

[56] L. Marisa, A. de Reyniès, A. Duval et al., "Gene expression classification of colon cancer into molecular subtypes: characterization, validation, and prognostic value," PLoS Medicine, vol. 10, no. 5, Article ID e1001453, 2013.

[57] N. J. Llosa, M. Cruise, A. Tam et al., "The vigorous immune microenvironment of microsatellite instable colon cancer is balanced by multiple counter-inhibitory checkpoints," Cancer Discovery, vol. 5, no. 1, pp. 43-51, 2015.

[58] E. Van Cutsem, R. Labianca, G. Bodoky et al., "Randomized phase III trial comparing biweekly infusional fluorouracil/ leucovorin alone or with irinotecan in the adjuvant treatment of stage III colon cancer: PETACC-3," Journal of Clinical Oncology, vol. 27, no. 19, pp. 3117-3125, 2009. 
[59] E. Fontana, K. Eason, A. Cervantes, R. Salazar, and A. Sadanandam, "Context matters-consensus molecular subtypes of colorectal cancer as biomarkers for clinical trials," Annals of Oncology, vol. 30, no. 4, pp. 520-527, 2019.

[60] H.-J. Lenz, F.-S. Ou, A.-P. Venook et al., "Impact of consensus molecular subtype on survival in patients with metastatic colorectal cancer: results from CALGB/SWOG 80405 (alliance)," Journal of Clinical Oncology, vol. 37, no. 22, pp. 1876-1885, 2019.

[61] S. Stintzing, P. Wirapati, H. J. Lenz, D. Neureiter, L. Fischer von Weikersthal, and T. Decker, "Consensus molecular subgroups (CMS) of colorectal cancer (CRC) and 1st-line efficacy of FOLFIRI plus cetuximab or bevacizumab in the FIRE3 (AIO KRK-0306) trial," Annals of Oncology, vol. 30, pp. 1796-1803, 2019.

[62] J. K. Mooi, P. Virapati, R. Asher et al., "The prognostic impact of consensus molecular subtypes (CMS) and its predictive effects for bevacizumab benefit in metastatic colorectal cancer: molecular analysis of the AGITG MAX clinical trial," Annals of Oncology, vol. 29, no. 11, pp. 2240-2246, 2018.

[63] L. Marisa, M. Ayadi, R. Balogoun et al., "Clinical utility of colon cancer molecular subtypes: validation of two main colorectal molecular classifications on the PETACC-8 phase III trial cohort," Journal of Clinical Oncology, vol. 35, no. 15 suppl, p. 3509, 2017.

[64] N. Song, K. L. Pogue-Geile, P. G. Gavin et al., "Clinical outcome from oxaliplatin treatment in stage II/III colon cancer according to intrinsic subtypes: secondary analysis of NSABP C-07/NRG oncology randomized clinical trial," JAMA Oncology, vol. 2, no. 9, p. 1162, 2016.

[65] N. Rodriguez-Salas, G. Dominguez, R. Barderas et al., "Clinical relevance of colorectal cancer molecular subtypes," Critical Reviews in Oncology and Hematology, vol. 109, pp. 9-19, 2017.

[66] R. V. Purcell, S. Schmeier, Y. C. Lau, J. F. Pearson, and F. A. Frizelle, "Molecular subtyping improves prognostication of Stage 2 colorectal cancer," BMC Cancer, vol. 19, no. 1, p. $1155,2019$.

[67] A. Sveen, J. Bruun, P. W. Eide et al., "Colorectal cancer consensus molecular subtypes translated to preclinical models uncover potentially targetable cancer cell dependencies," Clinical Cancer Research, vol. 24, no. 4, pp. 794-806, 2018.

[68] A. Okita, S. Takahashi, K. Ouchi et al., "Consensus molecular subtypes classification of colorectal cancer as a predictive factor for chemotherapeutic efficacy against metastatic colorectal cancer," Oncotarget, vol. 9, no. 27, 2018.

[69] "Modulation of fluorouracil by leucovorin in patients with advanced colorectal cancer: evidence in terms of response rate. advanced colorectal cancer meta-analysis project," Journal of Clinical Oncology, vol. 10, no. 6, pp. 896-903, 1992.

[70] J. Akkad, S. Bochum, and U. M. Martens, "Personalized treatment for colorectal cancer: novel developments and putative therapeutic strategies," Langenbeck's Archives of Surgery, vol. 400, no. 2, pp. 129-143, 2015.

[71] V. Loizzi, V. D. Vecchio, G. Gargano et al., "Biological pathways involved in tumor angiogenesis and bevacizumab based anti-angiogenic therapy with special references to ovarian cancer," International Journal of Molecular Sciences, vol. 18, no. 9, p. 1967, 2017.

[72] L. V. dos Santos, M. R. Cruz, G. de Lima Lopes, and J. P. d. S. N. Lima, "VEGF-A levels in bevacizumab-treated breast cancer patients: a systematic review and meta- analysis," Breast Cancer Research and Treatment, vol. 151, no. 3, pp. 481-489, 2015.

[73] J. M. Jürgensmeier, H.-J. Schmoll, J. D. Robertson et al., "Prognostic and predictive value of VEGF, sVEGFR-2 and CEA in mCRC studies comparing cediranib, bevacizumab and chemotherapy," British Journal of Cancer, vol. 108, no. 6, pp. 1316-1323, 2013.

[74] A. Lopez, K. Harada, M. Vasilakopoulou, N. Shanbhag, and J. A. Ajani, "Targeting angiogenesis in colorectal carcinoma," Drugs, vol. 79, no. 1, pp. 63-74, 2019.

[75] F. Kabbinavar, "Phase II, randomized trial comparing bevacizumab plus fluorouracil (FU)/Leucovorin (LV) with FU/ LV alone in patients with metastatic colorectal cancer," Journal of Clinical Oncology, vol. 21, no. 1, pp. 60-65, 2003.

[76] H. Hurwitz, L. Fehrenbacher, W. Novotny et al., "Bevacizumab plus irinotecan, fluorouracil, and leucovorin for metastatic colorectal cancer," New England Journal of Medicine, vol. 350, no. 23, pp. 2335-2342, 2004.

[77] L. B. Saltz, S. Clarke, E. Díaz-Rubio et al., "Bevacizumab in combination with oxaliplatin-based chemotherapy as firstline therapy in metastatic colorectal cancer: a randomized phase III study," Journal of Clinical Oncology, vol. 26, no. 12, pp. 2013-2019, 2008.

[78] G. P. Stathopoulos, "Treatment of colorectal cancer with and without bevacizumab: a phase III study," Oncology, vol. 78, no. 5-6, pp. 376-381, 2010.

[79] N. C. Tebbutt, K. Wilson, V. J. Gebski et al., "Capecitabine, bevacizumab, and mitomycin in first-line treatment of metastatic colorectal cancer: results of the australasian gastrointestinal trials group randomized phase III MAX study," Journal of Clinical Oncology, vol. 28, no. 19, pp. 3191-3198, 2010.

[80] C. S. Fuchs, J. Marshall, and J. Barrueco, "Randomized, controlled trial of irinotecan plus infusional, bolus, or oral fluoropyrimidines in first-line treatment of metastatic colorectal cancer: updated results from the BICC-C study," Journal of Clinical Oncology, vol. 26, no. 4, pp. 689-690, 2008.

[81] B. J. Giantonio, P. J. Catalano, N. J. Meropol et al., "Bevacizumab in combination with oxaliplatin, fluorouracil, and leucovorin (FOLFOX4) for previously treated metastatic colorectal cancer: results from the eastern cooperative oncology group study E3200," Journal of Clinical Oncology, vol. 25, no. 12, pp. 1539-1544, 2007.

[82] J. Bennouna, J. Sastre, D. Arnold et al., "Continuation of bevacizumab after first progression in metastatic colorectal cancer (ML18147): a randomised phase 3 trial," Lancet Oncology, vol. 14, no. 1, pp. 29-37, 2013.

[83] A. Patel and W. Sun, "Ziv-aflibercept in metastatic colorectal cancer," Biologics: Targets and Therapy, vol. 8, pp. 13-25, 2013.

[84] E. Van Cutsem, J. Tabernero, R. Lakomy et al., "Addition of aflibercept to fluorouracil, leucovorin, and irinotecan improves survival in a phase III randomized trial in patients with metastatic colorectal cancer previously treated with an oxaliplatin-based regimen," Journal of Clinical Oncology, vol. 30, no. 28, pp. 3499-3506, 2012.

[85] S. M. Wilhelm, J. Dumas, L. Adnane et al., "Regorafenib (BAY 73-4506): a new oral multikinase inhibitor of angiogenic, stromal and oncogenic receptor tyrosine kinases with potent preclinical antitumor activity," International Journal of Cancer, vol. 129, no. 1, pp. 245-255, 2011.

[86] A. Grothey, E. V. Cutsem, A. Sobrero et al., "Regorafenib monotherapy for previously treated metastatic colorectal cancer (CORRECT): an international, multicentre, 
randomised, placebo-controlled, phase 3 trial," The Lancet, vol. 381, no. 9863, pp. 303-312, 2013.

[87] M. Moore, S. Gill, T. Asmis et al., "Randomized phase II study of modified FOLFOX-6 in combination with ramucirumab or icrucumab as second-line therapy in patients with metastatic colorectal cancer after disease progression on first-line irinotecan-based therapy," Annals of Oncology, vol. 27, no. 12, pp. 2216-2224, 2016.

[88] R.-H. Xu, L. Shen, K.-M. Wang et al., "Famitinib versus placebo in the treatment of refractory metastatic colorectal cancer: a multicenter, randomized, double-blinded, placebocontrolled, phase II clinical trial," Chinese Journal of Cancer, vol. 36, no. 1, p. 97, 2017.

[89] J. Li, S. Qin, R.-H. Xu et al., "Effect of fruquintinib vs. placebo on overall survival in patients with previously treated metastatic colorectal cancer: the FRESCO randomized clinical trial," JAMA, vol. 319, no. 24, p. 2486, 2018.

[90] P. M. Hoff, A. Hochhaus, B. C. Pestalozzi et al., "Cediranib plus FOLFOX/CAPOX versus placebo plus FOLFOX/ CAPOX in patients with previously untreated metastatic colorectal cancer: a randomized, double-blind, phase III study (horizon II)," Journal of Clinical Oncology, vol. 30, no. 29, pp. 3596-3603, 2012.

[91] C. L. Arteaga and J. A. Engelman, "ERBB receptors: from oncogene discovery to basic science to mechanism-based cancer therapeutics," Cancer Cell, vol. 25, no. 3, pp. 282-303, 2014.

[92] C. Fortunato and T. Giampaolo, "EGFR antagonists in cancer treatment," New England Journal of Medicine, vol. 358, pp. 1160-1174, 2008.

[93] M. Scaltriti and J. Baselga, "The epidermal growth factor receptor pathway: a model for targeted therapy," Clinical Cancer Research, vol. 12, no. 18, pp. 5268-5272, 2006.

[94] A. E. Koyen, G. Nabeta, S. Bienfait, A. J. Schlafstein, D. S. Yu, and W. Daddacha, "EGFR role in cancer: a potential therapeutic target," in Role of Tyrosine Kinases in Gastrointestinal Malignancies, G. P. Nagaraju, Ed., Springer, Singapore, pp. 225-234, 2018.

[95] A. B. Singh, "EGFR-signaling and autophagy: how they fit in the cancer landscape," Journal of Adenocarcinoma, vol. 1, no. 2, 2016.

[96] S. Sigismund, D. Avanzato, and L. Lanzetti, "Emerging functions of the EGFR in cancer," Molecular Oncology, vol. 12, no. 1, pp. 3-20, 2018.

[97] H. Masui, T. Kawamoto, J. D. Sato, B. Wolf, G. Sato, and J. Mendelsohn, "Growth inhibition of human tumor cells in athymic mice by anti-epidermal growth factor receptor monoclonal antibodies," Journal of Urology, vol. 132, no. 5, p. $1060,1984$.

[98] D. Cunningham, Y. Humblet, S. Siena et al., "Cetuximab monotherapy and cetuximab plus irinotecan in irinotecanrefractory metastatic colorectal cancer," New England Journal of Medicine, vol. 351, no. 4, pp. 337-345, 2004.

[99] E. V. Cutsem, C.-H. Köhne, E. Hitre et al., "Cetuximab and chemotherapy as initial treatment for metastatic colorectal cancer," New England Journal of Medicine, vol. 360, pp. 1408-1417, 2009.

[100] C. Bokemeyer, I. Bondarenko, A. Makhson et al., "Fluorouracil, leucovorin, and oxaliplatin with and without cetuximab in the first-line treatment of metastatic colorectal cancer," Journal of Clinical Oncology, vol. 27, pp. 663-671, 2009.

[101] C. Bokemeyer, E. V. Custem, P. Rougier et al., "Addition of cetuximab to chemotherapy as first-line treatment for KRAS wild-type metastatic colorectal cancer: pooled analysis of the CRYSTAL and OPUS randomised clinical trials," European Journal of Cancer, vol. 48, no. 10, pp. 1466-1475, 2012.

[102] S. Kopetz, A. Grothey, R. Yaeger et al., "Encorafenib, binimetinib, and cetuximab in BRAF V600E-mutated colorectal cancer," New England Journal of Medicine, vol. 381, no. 17, pp. 1632-1643, 2019.

[103] A. F. Sobrero, J. Maurel, L. Fehrenbacher et al., "EPIC: phase III trial of cetuximab plus irinotecan after fluoropyrimidine and oxaliplatin failure in patients with metastatic colorectal cancer," Journal of Clinical Oncology, vol. 26, pp. 2311-2319, 2008.

[104] C. S. Karapetis, S. Khambata-Ford, D. J. Jonker et al., "K-ras mutations and benefit from cetuximab in advanced colorectal cancer," New England Journal of Medicine, vol. 359, no. 17, pp. 1757-1765, 2008.

[105] M. Cohenuram and M. W. Saif, "Panitumumab the first fully human monoclonal antibody: from the bench to the clinic:," Anti-Cancer Drugs, vol. 18, no. 1, pp. 7-15, 2007.

[106] E. V. Cutsem, M. Peeters, S. Siena et al., “Open-label phase III trial of panitumumab plus best supportive care compared with best supportive care alone in patients with chemotherapy-refractory metastatic colorectal cancer," Journal of Clinical Oncology, vol. 25, no. 13, pp. 1658-1664, 2007.

[107] J. Y. Douillard, S. Siena, J. Cassidy et al., "Final results from PRIME: randomized phase 3 study of panitumumab with FOLFOX4 for first-line treatment of metastatic colorectal cancer," Annals of Oncology, vol. 25, pp. 1346-1355, 2014.

[108] M. Peeters, T. Jay Price, A. Cervantes et al., "Randomized phase III study of panitumumab with fluorouracil, leucovorin, and irinotecan (FOLFIRI) compared with FOLFIRI alone as second-line treatment in patients with metastatic colorectal cancer," Journal of Clinical Oncology, vol. 28, no. 31, pp. 4706-4713, 2010.

[109] S. Corso and S. Giordano, "Targeted therapies in cancer and mechanisms of resistance," Journal of Molecular Medicine, vol. 92, no. 7, pp. 677-679, 2014.

[110] V. Sforza, E. Martinelli, F. Ciardiello et al., "Mechanisms of resistance to anti-epidermal growth factor receptor inhibitors in metastatic colorectal cancer," World Journal of Gastroenterology, vol. 22, no. 28, p. 6345, 2016.

[111] A. Karani, T. C. Felismino, L. Diniz, M. Petaccia Macedo, V. Souza e Silva, and C. Abdon Mello, "Is there a role for rechallenge and reintroduction of anti-EGFR plus chemotherapy in later lines of therapy for metastatic colorectal carcinoma? A retrospective analysis," Ecancermedicalscience, vol. 14, 2020.

[112] S. Misale, F. Di Nicolantonio, A. Sartore-Bianchi, S. Siena, and A. Bardelli, "Resistance to anti-EGFR therapy in colorectal cancer: from heterogeneity to convergent evolution," Cancer Discovery, vol. 4, no. 11, pp. 1269-1280, 2014.

[113] B. Zhao, L. Wang, H. Qui et al., "Mechanisms of resistance to anti-EGFR therapy in colorectal cancer," Oncotarget, vol. 8, no. 3, pp. 3980-4000, 2017.

[114] V. K. Morris, F. A. San Lucas, M. J. Overman et al., "Clinicopathologic characteristics and gene expression analyses of non-KRAS 12/13, RAS-mutated metastatic colorectal cancer," Annals of Oncology, vol. 25, no. 10, pp. 2008-2014, 2014

[115] E. V. Cutsem, I. Lang, G. D’haens et al., "KRAS status and efficacy in the first-line treatment of patients with metastatic colorectal cancer (mCRC) treated with FOLFIRI with or without cetuximab: the CRYSTAL experience," Journal of Clinical Oncology, vol. 26, no. 15_suppl, p. 2, 2008. 
[116] C. Bokemeyer, I. Bondarenko, J. T. Hartmann et al., "KRAS status and efficacy of first-line treatment of patients with metastatic colorectal cancer (mCRC) with FOLFOX with or without cetuximab: the OPUS experience," Journal of Clinical Oncology, vol. 26, no. 15_suppl, p. 4000, 2008.

[117] C. J. Allegra, J. M. Jessup, M. R. Somerfield et al., “American Society of Clinical Oncology provisional clinical opinion: testing for KRAS gene mutations in patients with metastatic colorectal carcinoma to predict response to anti-epidermal growth factor receptor monoclonal antibody therapy," Journal of Clinical Oncology, vol. 27, no. 12, pp. 2091-2096, 2009.

[118] N. Piton, F. Borrini, A. Bolognese, A. Lamy, and J.-C. Sabourin, "KRAS and BRAF mutation detection: is immunohistochemistry a possible alternative to molecular biology in colorectal cancer?" Gastroenterology Research and Practice, vol. 2015, Article ID 753903, 8 pages, 2015.

[119] F. Loupakis, A. Ruzzo, C. Cremolini et al., "KRAS codon 61, 146 and BRAF mutations predict resistance to cetuximab plus irinotecan in KRAS codon 12 and 13 wild-type metastatic colorectal cancer," British Journal of Cancer, vol. 101, pp. 715-721, 2009.

[120] C. Bokemeyer, C.-H. Kohne, F. Ciardiello et al., "Treatment outcome according to tumor RAS mutation status in OPUS study patients with metastatic colorectal cancer (mCRC) randomized to FOLFOX4 with/without cetuximab," Journal of Clinical Onclogy, vol. 20, 2014.

[121] F. Ciardiello, H.-J. Lenz, C.-H. Kohne et al., “Treatment outcome according to tumor RAS mutation status in CRYSTAL study patients with metastatic colorectal cancer (mCRC) randomized to FOLFIRI with/without cetuximab," Journal of Clinical Onclogy, vol. 32, no. 15_suppl, p. 3506, 2014.

[122] J.-Y. Douillard, K. S. Oliner, S. Siena et al., "PanitumumabFOLFOX4 treatment and RAS mutations in colorectal cancer," New England Journal of Medicine, vol. 369, no. 11, pp. 1023-1034, 2013.

[123] H. Davies, G. R. Bignell, C. Cox et al., "Mutations of the BRAF gene in human cancer," Nature, vol. 417, no. 6892, pp. 949-954, 2002.

[124] Z. Saridaki, M. Tzardi, M. Sfakianaki et al., "BRAFV600E mutation analysis in patients with metastatic colorectal cancer (mCRC) in daily clinical practice: correlations with clinical characteristics, and its impact on patients' outcome," PLoS One, vol. 8, no. 12, Article ID e84604, 2013.

[125] D. P. Modest, A. Jung, N. Moosmann et al., "The influence of KRAS and BRAF mutations on the efficacy of cetuximabbased first-line therapy of metastatic colorectal cancer: an analysis of the AIO KRK-0104-trial," International Journal of Cancer, vol. 131, no. 4, pp. 980-986, 2012.

[126] Q. Xu, A. T. Xu, M. M. Zhu, J. L. Tong, X. T. Xu, and Z. H. Ran, "Predictive and prognostic roles of BRAF mutation in patients with metastatic colorectal cancer treated with anti-epidermal growth factor receptor monoclonal antibodies: a meta-analysis: predictive and prognostic roles of BRAF," Journal of Digestive Diseases, vol. 14, no. 8, pp. 409-416, 2013.

[127] A. Rowland, M. M. Dias, M. D. Wiese et al., "Meta-analysis of BRAF mutation as a predictive biomarker of benefit from anti-EGFR monoclonal antibody therapy for RAS wild-type metastatic colorectal cancer," British Journal of Cancer, vol. 112, no. 12, pp. 1888-1894, 2015.

[128] S. A. Danielsen, P. W. Eide, A. Nesbakken, T. Guren, E. Leithe, and R. A. Lothe, "Portrait of the PI3K/AKT pathway in colorectal cancer," Biochimica et Biophysica Acta, vol. 1855, no. 1, pp. 104-121, 2015.

[129] J. A. Engelman, "Targeting PI3K signalling in cancer: opportunities, challenges and limitations," Nature Reviews Cancer, vol. 9, no. 8, pp. 550-562, 2009.

[130] A. Sartore-Bianchi, M. Martini, F. Molinari et al., "PIK3CA mutations in colorectal cancer are associated with clinical resistance to EGFR-targeted monoclonal antibodies," Cancer Research, vol. 69, no. 5, pp. 1851-1857, 2009.

[131] W. De Roock, B. Claes, D. Bernasconi et al., "Effects of KRAS, BRAF, NRAS, and PIK3CA mutations on the efficacy of cetuximab plus chemotherapy in chemotherapy-refractory metastatic colorectal cancer: a retrospective consortium analysis," Lancet Oncology, vol. 11, no. 8, pp. 753-762, 2010.

[132] C. Mao, Z. Y. Yang, X. F. Hu, Q. Chen, and J. L. Tang, "PIK3CA exon 20 mutations as a potential biomarker for resistance to anti-EGFR monoclonal antibodies in KRAS wild-type metastatic colorectal cancer: a systematic review and meta-analysis," Annals of Oncology, vol. 23, no. 6, pp. 1518-1525, 2012.

[133] V. Stambolic, A. Suzuki, J. L. de la Pompa et al., "Negative regulation of $\mathrm{PKB} /$ Akt-dependent cell survival by the tumor suppressor PTEN,” Cell, vol. 95, no. 1, pp. 29-39, 1998.

[134] F. Loupakis, L. Pollina, I. Stasi et al., "PTEN expression and KRAS mutations on primary tumors and metastases in the prediction of benefit from cetuximab plus irinotecan for patients with metastatic colorectal cancer," Journal of Clinical Oncology, vol. 27, no. 16, pp. 2622-2629, 2009.

[135] M. Frattini, P. Saletti, E. Romagnani et al., "PTEN loss of expression predicts cetuximab efficacy in metastatic colorectal cancer patients," British Journal of Cancer, vol. 97, no. 8, pp. 1139-1145, 2007.

[136] Z.-H. Wang, Q.-Y. Gao, and J.-Y. Fang, "Loss of PTEN expression as a predictor of resistance to anti-EGFR monoclonal therapy in metastatic colorectal cancer: evidence from retrospective studies," Cancer Chemotherapy and Pharmacology, vol. 69, no. 6, pp. 1647-1655, 2012.

[137] E. Razis, E. Briasoulis, E. Vrettou et al., "Potential value of PTEN in predicting cetuximab response in colorectal cancer: an exploratory study," BMC Cancer, vol. 8, no. 1, p. 234, 2008.

[138] P. Laurent-Puig, A. Cayre, G. Manceau et al., "Analysis of PTEN, BRAF, and EGFR status in determining benefit from cetuximab therapy in wild-type KRAS metastatic colon cancer," Journal of Clinical Oncology, vol. 27, no. 35, pp. 5924-5930, 2009.

[139] E. I. Ágoston, T. Micsik, B. Ács et al., "In depth evaluation of the prognostic and predictive utility of PTEN immunohistochemistry in colorectal carcinomas: performance of three antibodies with emphasis on intracellular and intratumoral heterogeneity," Diagnostic Pathology, vol. 11, no. 1, p. 61, 2016.

[140] L. Salvatore, M. A. Calegari, F. Loupakis et al., "PTEN in colorectal cancer: shedding light on its role as predictor and target," Cancers, vol. 11, no. 11, p. 1765, 2019.

[141] A. Stahler, S. Stintzing, D. P. Modest et al., "Amphiregulin expression is a predictive biomarker for EGFR inhibition in metastatic colorectal cancer: combined analysis of three randomized trials," Clinical Cancer Research, vol. 26, pp. 6559-6567, 2020.

[142] J. F. Seligmann, F. Elliott, S. D. Richman et al., "Combined epiregulin and amphiregulin expression levels as a predictive biomarker for panitumumab therapy benefit or lack of 
benefit in patients with RAS wild-type advanced colorectal cancer," JAMA Oncology, vol. 2, no. 5, p. 633, 2016.

[143] P. Llovet, J. Sastre, J. S. Ortega et al., "Prognostic value of BRAF, PI3K, PTEN, EGFR copy number, amphiregulin and epiregulin status in patients with KRAS codon 12 wild-type metastatic colorectal cancer receiving first-line chemotherapy with anti-EGFR therapy," Molecular Diagnosis and Therapy, vol. 19, no. 6, pp. 397-408, 2015.

[144] M. Yoshida, T. Shimura, M. Sato et al., "A novel predictive strategy by immunohistochemical analysis of four EGFR ligands in metastatic colorectal cancer treated with antiEGFR antibodies," Journal of Cancer Research and Clinical Oncology, vol. 139, no. 3, pp. 367-378, 2013.

[145] S. Khambata-Ford, C. R. Garrett, N. J. Meropol et al., "Expression of epiregulin and amphiregulin and K-ras mutation status predict disease control in metastatic colorectal cancer patients treated with cetuximab," Journal of Clinical Oncology, vol. 25, no. 22, pp. 3230-3237, 2007.

[146] B. Jacobs, W. De Roock, H. Piessevaux et al., "Amphiregulin and epiregulin mRNA expression in primary tumors predicts outcome in metastatic colorectal cancer treated with cetuximab," Journal of Clinical Oncology, vol. 27, no. 30, pp. 5068-5074, 2009.

[147] F. Bormann, S. Stinzing, S. Tierling et al., "Epigenetic regulation of amphiregulin and epiregulin in colorectal cancer," International Journal of Cancer, vol. 144, no. 3, pp. 569-581, 2019.

[148] F. Laudisi, F. Cherubini, G. Monteleone, and C. Stolfi, "STAT3 interactors as potential therapeutic targets for cancer treatment," International Journal of Molecular Sciences, vol. 19, no. 6, p. 1787, 2018.

[149] F. M. Corvinus, C. Orth, R. Moriggl et al., "Persistent STAT3 activation in colon cancer is associated with enhanced cell proliferation and tumor growth," Neoplasia, vol. 7, no. 6, pp. 545-555, 2005.

[150] E. Dobi, F. Monnien, S. Kim et al., "Impact of STAT3 phosphorylation on the clinical effectiveness of anti-EGFRbased therapy in patients with metastatic colorectal cancer," Clinical Colorectal Cancer, vol. 12, no. 1, pp. 28-36, 2013.

[151] N. Ung, T. L. Putoczki, S. S. Stylli et al., "Anti-EGFR therapeutic efficacy correlates directly with inhibition of STAT3 activity," Cancer Biology and Therapy, vol. 15, no. 5, pp. 623-632, 2014.

[152] B. O. Van Emburgh, A. Sartore-Bianchi, F. Di Nicolantonio, S. Siena, and A. Bardelli, "Acquired resistance to EGFRtargeted therapies in colorectal cancer," Molecular Oncology, vol. 8, no. 6, pp. 1084-1094, 2014.

[153] L. A. Diaz Jr., R. T. Williams, J. Wu et al., "The molecular evolution of acquired resistance to targeted EGFR blockade in colorectal cancers," Nature, vol. 486, no. 7404, pp. 537-540, 2012.

[154] S. Misale, R. Yaeger, S. Hobor et al., "Emergence of KRAS mutations and acquired resistance to anti-EGFR therapy in colorectal cancer," Nature, vol. 486, no. 7404, pp. 532-536, 2012.

[155] C. Montagut, A. Dalmases, B. Bellosillo et al., "Identification of a mutation in the extracellular domain of the epidermal growth factor receptor conferring cetuximab resistance in colorectal cancer," Nature Medicine, vol. 18, no. 2, pp. 221-223, 2012.

[156] C. Esposito, A. M. Rachiglio, M. L. L. Porta et al., "The S492R EGFR ectodomain mutation is never detected in KRAS wildtype colorectal carcinoma before exposure to EGFR monoclonal antibodies," Cancer Biology and Therapy, vol. 14, no. 12, pp. 1143-1146, 2013.

[157] T. Price, A. Ang, M. Boedigheimer et al., "Frequency of S492R mutations in the epidermal growth factor receptor: analysis of plasma DNA from patients with metastatic colorectal cancer treated with panitumumab or cetuximab monotherapy," Cancer Biology and Therapy, vol. 21, pp. 891-898, 2020.

[158] K. Yonesaka, K. Zejnullahu, I. Okamoto et al., "Activation of ERBB2 signaling causes resistance to the EGFR-directed therapeutic antibody cetuximab," Science Translational Medicine, vol. 3, no. 99, 2011.

[159] A. Bertotti, G. Migliardi, F. Galimi et al., "A molecularly annotated platform of patient-derived xenografts ("xenopatients") identifies HER2 as an effective therapeutic target in cetuximab-resistant colorectal cancer," Cancer Discovery, vol. 1, no. 6, pp. 508-523, 2011.

[160] V. Martin, L. Landi, F. Molinari et al., "HER2 gene copy number status may influence clinical efficacy to anti-EGFR monoclonal antibodies in metastatic colorectal cancer patients," British Journal of Cancer, vol. 108, no. 3, pp. 668-675, 2013.

[161] W. Ariyawutyakorn, S. Saichaemchan, and M. VarellaGarcia, "Understanding and targeting MET signaling in solid tumors-are we there yet?" Journal of Cancer, vol. 7, no. 6, pp. 633-649, 2016.

[162] D. Liska, C.-T. Chen, T. Bachleitner-Hofmann, J. G. Christensen, and M. R. Weiser, "HGF rescues colorectal cancer cells from EGFR inhibition via MET activation," Clinical Cancer Research, vol. 17, no. 3, pp. 472-482, 2011.

[163] A. Bardelli, S. Corso, A. Bertotti et al., "Amplification of the MET receptor drives resistance to anti-EGFR therapies in colorectal cancer," Cancer Discovery, vol. 3, no. 6, pp. 658-673, 2013.

[164] T. Troiani, E. Martinelli, S. Napolitano et al., "Increased TGF- as a mechanism of acquired resistance to the antiEGFR inhibitor cetuximab through EGFR-MET interaction and activation of MET signaling in colon cancer cells," Clinical Cancer Research, vol. 19, no. 24, pp. 6751-6765, 2013.

[165] F. Galimi, D. Torti, F. Sassi et al., "Genetic and expression analysis of MET, MACC1, and HGF in metastatic colorectal cancer: response to met inhibition in patient xenografts and pathologic correlations," Clinical Cancer Research, vol. 17, no. 10, pp. 3146-3156, 2011.

[166] N. C. Turner and J. S. Reis-Filho, "Genetic heterogeneity and cancer drug resistance," Lancet Oncology, vol. 13, no. 4, pp. e178-e185, 2012. 